\title{
Utility of miR-133a-3p as a diagnostic indicator for hepatocellular carcinoma: An investigation combined with GEO, TCGA, meta-analysis and bioinformatics
}

\author{
HAI-WEI LIANG $^{1 *}$, XIA YANG $^{1 *}$, DONG-YUE WEN ${ }^{2}$, LI GAO $^{1}$, XIANG-YU ZHANG ${ }^{1}$, \\ ZHI-HUA YE ${ }^{1}$, JIE LUO ${ }^{3}$, ZU-YUN LI ${ }^{2}$, YUN HE ${ }^{2}$, YU-YAN PANG ${ }^{1}$ and GANG CHEN ${ }^{1}$ \\ Departments of ${ }^{1}$ Pathology, ${ }^{2}$ Medical Ultrasonics and ${ }^{3}$ Medical Oncology, The First Affiliated Hospital of \\ Guangxi Medical University, Nanning, Guangxi Zhuang Autonomous Region 530021, P.R. China
}

Received November 12, 2016; Accepted June 27, 2017

DOI: $10.3892 / \mathrm{mmr} .2017 .8040$

\begin{abstract}
Increasing evidence has demonstrated that microRNA (miR)-133a-3p is an important regulator of hepatocellular carcinoma (HCC). In the present study, the diagnostic role of miR-133a-3p in HCC, and the potential functional pathways, were both explored based on publicly available data. Eligible microarray datasets were collected from NCBI Gene Expression Omnibus (GEO) database and ArrayExpress database. The data related to HCC and matched adjacent normal tissues were also downloaded from The Cancer Genome Atlas (TCGA). Published studies reporting the association between miR-133a-3p expression and HCC were reviewed from multiple databases. By combining the data derived from three sources (GEO, TCGA and published studies), the authors analyzed the comprehensive relationship between miR-133a-3p expression and clinicopathological features of HCC. Eventually, putative targets of miR-133a-3p in HCC were selected for further bioinformatics prediction. A total of eight published microarray datasets were gathered, and the pooled results demonstrated that the expression of miR-133a-3p in the tumor group was lower than that in normal groups [standardized mean difference $(\mathrm{SMD})=-0.54 ; 95 \%$ confidence interval (CI), -0.74 to -0.35 ; $\mathrm{P}<0.001]$. Consistently, the level
\end{abstract}

Correspondence to: Dr Yun He, Department of Medical Ultrasonics, The First Affiliated Hospital of Guangxi Medical University, 6 Shuangyong Road, Nanning, Guangxi Zhuang Autonomous Region 530021, P.R. China

E-mail: 228388072@qq.com

Dr Yu-Yan Pang, Department of Pathology, The First Affiliated Hospital of Guangxi Medical University, 6 Shuangyong Road, Nanning, Guangxi Zhuang Autonomous Region 530021, P.R. China E-mail: hornor159@126.com

*Contributed equally

Key words: meta-analysis, microRNA-133a-3p, hepatocellular carcinoma, bioinformatics, Gene Expression Omnibus, TCGA of miR-133a-1 in HCC was reduced markedly compared to normal tissues $(\mathrm{P}<0.001)$ based on TCGA data, and the AUC value of low miR-133a-1 expression for HCC diagnosis was $0.670(\mathrm{P}<0.001)$. Furthermore, the combined SMD of all datasets (GEO, TCGA and literature) suggested that significant difference was observed between the HCC group and the normal control group, and lower miR-133a-3p expression in HCC group was noted (SMD=-0.69; 95\% CI, -1.10 to -0.29 ; $\mathrm{P}=0.001)$. In addition, the authors discovered five key genes of the calcium signaling pathway (NOS1, ADRA1A, ADRA1B, ADRA1D and TBXA2R) that may probably be targeted by miR-133a-3p in HCC. The study reveals that miR-133a-3p may function as a tumor suppressor in HCC. The prospective novel pathways and key genes of miR-133a-3p could offer potential biomarkers for $\mathrm{HCC}$; however, the predictions require further confirmation.

\section{Introduction}

Hepatocellular carcinoma (HCC) is one of the most common and lethal malignancies in the world with extremely poor prognosis. HCC is also the second top cause of cancer-related death and its incidence ranked fifth in diagnosed cancers globally (1). Moreover, HCC is highly prevalent in many Asian countries owing to its high endemicity of hepatitis B virus (HBV) infection, especially in China (2-4). Multiple treatment possibilities are available for HCC, such as curative resection, liver transplantation, radiofrequency ablation, systemic targeted agents like sorafenib, whereas the management of advanced HCC still presents as a therapeutic challenge over the years (5-9). Despite these developments in surgery and other strategies, the prognosis of HCC patients is still unsatisfactory due to the non-curable stages at the time of diagnosis (10-14). Therefore, early diagnosis of HCC is expected and the molecular pathogenesis of HCC needs to be better elucidated.

MicroRNAs (miRNAs) are a class of highly conserved, endogenous, small non-coding RNA molecules of 18-23 nucleotides in length, which act as post-transcriptional regulators by binding to 3'-untranslated regions (3'-UTR) of mRNA for target genes (15-19). In recent years, researchers have focused on the importance of miRNAs in carcinogenesis (20-22). A 
large amount of evidence has suggested that miRNAs may act as oncogenes or tumor suppressors by adapting pathophysiological progressions (23-27). In the new era of cancer therapy, miRNAs are anticipated for early diagnosis and treatment of patients in order to prolong their survival time (28-31).

miR-133a-3p, which belongs to the miR-133 family, was first experimentally characterized in mice (32). In the human genome, miR-133a-3p is a multicopy gene, with two known copies: miR-133a-1 and miR-133a-2, located on chromosomes 18, 20 respectively. To date, multiple functional roles of miR-133a-3p have been elucidated, such as regulating myoblast proliferation and differentiation (33), inhibiting embryonic cardiomyocyte proliferation (34) and avoiding genetic cardiac hypertrophy (35). Furthermore, it has been reported that miR-133a-3p is among the most frequently downregulated miRNAs in various types of human malignancies, which suggested that miR-133a-3p may serve a critical part in tumor progression of various malignancies, including non-small cell lung cancer (36), ovarian cancer (37), colorectal cancer (38), bladder cancer (39), breast cancer (40) and prostate cancer (41). Nevertheless, there are rare reports focusing on the role of miR-133a-3p in HCC. The results of miR-133a-3p profile detection in $\mathrm{HCC}$ compared to non-tumor liver tissues by microarray technologies, reverse transcription-quantitative polymerase chain reaction and high throughput technologies are contradictory. Moreover, previous individual conclusion may not be reliable due to the small sample size. Further explorations of the clinical role and the molecular mechanism of miR-133a-3p in HCC are urgently needed.

Hence, the authors investigated the expression of miR-133a-3p in tissues of HCC patients to illuminate its value as a diagnostic biomarker based on data from Gene Expression Omnibus (GEO), ArrayExpress, The Cancer Genome Atlas (TCGA) and literature. In addition, the authors studied these data sets with an integrated bioinformatics framework to explore how miR-133a-3p prospectively performs its function in HCC. The pipeline of the present study is charted in Fig. 1.

\section{Materials and methods}

\section{Dataset sources}

GEO and ArrayExpress database searching. Firstly, the microarrays related to HCC were obtained from GEO database (www.ncbi.nlm.gov/geo) and ArrayExpress (www.ebi. ac.uk/arrayexpress). The search terms included 'malignant' or 'cancer' or 'tumor' or 'tumour' or 'neoplasm' or 'carcinoma' and 'hepatocellular or liver or hepatic or HCC'. Then, microarrays that contained miR-133a-3p probe were screened for further analysis (Fig. 2).

TCGA data including 377 samples and 50 normal tissues downloading. The publicly available miRNA-seq and RNA-seq data of the miRNA and mRNA level of Liver hepatocellular carcinoma (LIHC) samples were obtained directly from the TCGA data portal (https://tcga-data.nci.nih.gov/tcga/tcgaHome2.jsp) via bulk download mode [LIHC (cancer type), miRNASeqV2 or RNASeqV2 (data type), level 3 (data level), All (preservation) and 1.12.0 (data version)] on October 16, 2016. The data were sequenced based on Illumina Genome Analyzer miRNA or RNA Sequencing platform. Gene expression data from miRNASeqV2 or RNASeqV2 results were quantified by Expectation-Maximization (RSEM) $(42,43)$ with the 'rsem. gene.normalized_results' file type. Extracted data were applied with no further transformation, except by rounding off values to integers. These downloaded data included a total of 377 HCC samples and 50 normal liver samples. The data of miR-133a were extracted and analyzed. The differentially expressed genes (DEGs) between the HCC and the normal liver control samples were identified by calculating the level of fold change (FC) in $\mathrm{HCC}$ vs. normal liver tissue with $\mathrm{R}$. The genes with a FC value $>2$ or $<0.5$ and with $\mathrm{P}<0.05$ were analyzed with Student's t-test were selected as DEGs in the present study.

Literature reviewing. Studies published before October 16, 2016 that reported the relationship between miR-133a-3p expression and HCC were retrieved from PubMed, Web of Science, EMBASE, Wiley Online Library, Cochrane Central Register of Controlled Trials, Science Direct, Google Scholar, Ovid, LILACS, Chinese CNKI, Chong Qing VIP, Wan Fang and China Biology Medicine disc. The searching terms were as follows: ('malignant' or 'cancer' or 'tumor' or 'tumour' or 'neoplasm' or 'carcinoma') and ('hepatocellular or liver or hepatic or HCC') and (miR-133a or miRNA-133a or microRNA-133a or miR133a or miRNA133a or microRNA133a or 'miR 133a' or 'miRNA $133 a$ ' or 'microRNA $133 a$ ' or miR-133a-3p or miRNA-133a-3p or microRNA-133a-3p or miR133a-3p or miRNA133a-3p or microRNA133a-3p or 'miR 133a-3p' or 'miRNA 133a-3p' or 'microRNA 133a-3p').

Study identification. Studies including microarrays or publications were considered eligible if they met following criteria: i) Study on human HCC tissues; ii) miR-133a-3p expression in tissues was measured and analyzed; iii) literatures were published in Chinese or English; iv) when data of the same patients were published in more than one article, only the latest publication was included; and v) as to microarrays, both HCC patients and normal samples were included in each dataset, and each group contained more than two samples.

Studies were excluded under the following criteria: i) Letters, case reports, reviews, or conference reports were excluded; ii) the required data could not be extracted or calculated from the original article; iii) the article was not found in full or had been published repeatedly; and iv) in vitro studies.

Data extraction. All the eligible studies were carefully reviewed and then the information was extracted from the inclusive studies independently by two investigators (Hai-Wei Liang and Xia Yang). Disagreements were negotiated by a third investigator (Gang Chen) and agreements were reached by discussion. With the included criteria, the following characteristics were obtained from eligible articles: First author's name or series (prefix, GSE) accession number, year of publication, country, number of patients and means and standard deviations of miR-133a-3p expression level.

Prediction of targeted genes of miR-133a-3p. An online prediction software miRWalk, which included 12 silico databases [such as TargetScan (www.targetscan.org), miRanda (www.miRnada.org) and PicTar (pictar.mdc-berlin.de)] was utilized to acquire the potential target mRNAs of miR-133a-3p. 


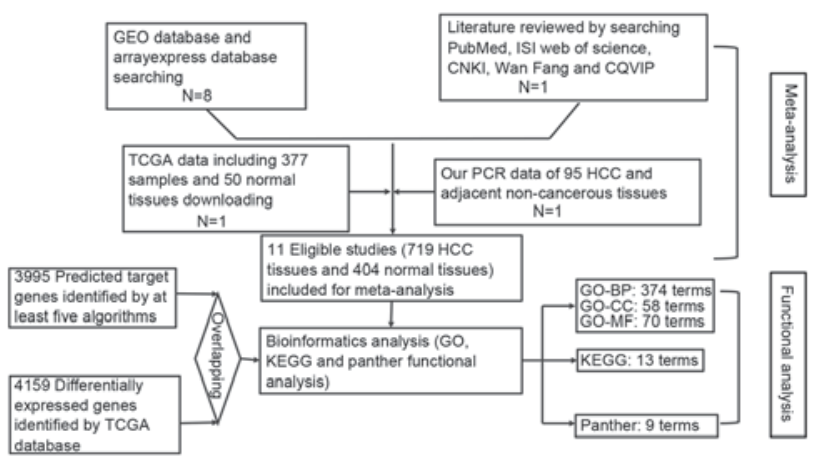

Figure 1. Schematic diagram depicting the analysis pipeline of the whole study. GEO, Gene Expression Omnibus; TCGA, The Cancer Genome Atlas; GO, Gene Ontology; BP, biological processes; CC, cellular components; MF molecular functions; KEGG, Kyoto Encyclopedia of Genes and Genomes.

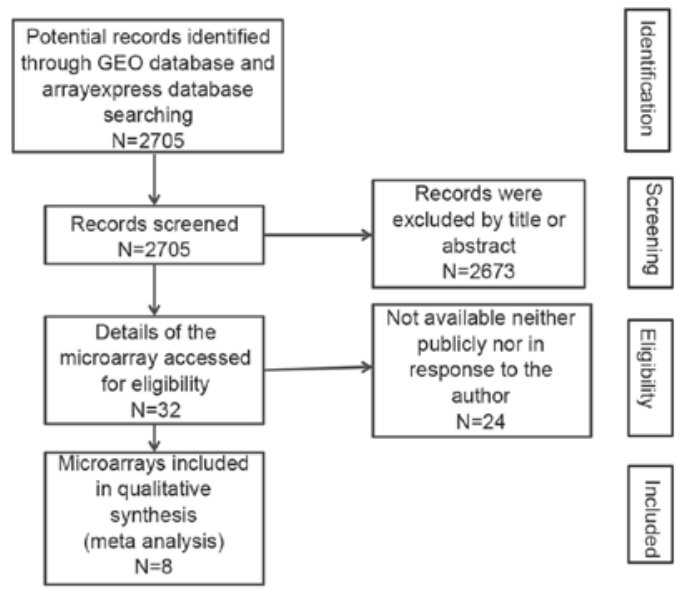

Figure 2. The microarray chips screening flow chart. Datasets included for meta-analysis were screened through GEO database and ArrayExpress database. GEO, Gene Expression Omnibus.

A total of 3,995 target genes were predicted initially, and only those genes appearing in more than five prediction software programs were essential for the present study.

Functional analyses. To further explore the biological processes and signal pathways in which miR-133a-3p could be involved and also to provide a theoretical foundation for future studies, functional analyses were performed subsequently. Gene Ontology (GO) analysis was conducted, since it demonstrates the predominant functions of the target genes from three aspects: Biological process, molecular function, as well as cellular component. Kyoto Encyclopedia of Genes and Genomes (KEGG) pathway analysis (www.genome.jp/kegg) was used to excavate remarkable pathways associated with target genes. All GO, KEGG pathway and panther analyses were performed to gain a further insight into the function of these potential target genes of miR-133a-3p in HCC by database for Annotation, Visualization and Integrated Discovery (DAVID). $\mathrm{P}<0.01$ for $\mathrm{GO}$ terms and $\mathrm{P}<0.05$ for pathways were considered statistically significant.

Protein- protein interactions (PPIs) network construction. The potential target genes in the top terms of KEGG analysis were employed to construct PPIs network by STRING 10.0 (http://string-db.org/), a Search Tool for the Retrieval of Interacting Genes/Proteins, with the minimum required interaction score 0.4 (44). The related data were downloaded from Tables/Exports module and the complete PPI network was drawn, then hub genes were identified. The proteins interactions information in STRING was derived from four sources, i.e., i) literature-reported protein interactions; ii) high-throughput experiments; iii) genome analysis and prediction; and iv) co-expression studies. The protein product of a gene serves as a node in the PPI network, and the connectivity degree denotes the interplayed protein numbers of the specific protein.

Statistical analysis. As for data obtained from the TCGA database, the miR-133a expression values were presented in a way as means \pm standard deviation. Receiver operating characteristic (ROC) curves were performed to judge the diagnostic value of miR-133a in HCC. The area under the curve (AUC) and P-value were calculated. Student's t-test was applied to verify the difference between two corresponding groups of various clinical features. Box-plots were drawn when significant difference existed in two compared ones. All above analyses were carried out via SPSS software (version 22.0; IBM, SPSS, Armonk, NY, USA). With respect to meta-analysis, standardized mean difference (SMD) and 95\% confidence interval (CI) were counted for pooled values. Sensitivity analysis was conducted to assess the stability of included studies. Cochran's $\mathrm{Q}$ and $\mathrm{I}^{2}$ test were used to evaluate heterogeneity, and $\mathrm{P}<0.1$ indicated significant heterogeneity. Publication bias was estimated by the Egger's and Begg's test. Meta-analysis was conducted using STATA 12.0 (StataCorp LLC, College Station, TX, USA). P $<0.05$ was considered to indicate a statistically significant difference.

\section{Results}

Signatures of GEO datasets and meta-analysis. The authors first searched GEO datasets to assess the expression of miR-133a-3p between HCC and non-cancer tissues. miR-133a-3p levels in eight datasets GSE57555 (Japan), GSE69580 (Taiwan), GSE54751 (USA), GSE41874 (Japan), GSE40744 (USA), GSE21362 (Japan), GSE22058 (USA) and GSE12717 (USA) which included $217 \mathrm{HCC}$ and 219 normal tissues are presented in Fig. 3. One dataset about recurrence (GSE64989, Germany) and two for invasiveness (GSE67138; GSE67139, USA) were also presented. Then meta-analysis containing eight microarrays was conducted to evaluate the diagnostic value of miR-133a-3p in HCC. SMD with 95\% CIs from involved datasets were pooled, and default fixed-effects model was first used. The pooled results revealed that noticeable difference was observed between tumor and normal groups ( $\mathrm{SMD}=-0.54 ; 95 \% \mathrm{CI},-0.74$ to $-0.35 ; \mathrm{P}<0.001 ;$ Fig. 4). No significant heterogeneity was found $\left(\mathrm{I}^{2}=36.5 \%\right.$; $\left.\mathrm{P}=0.138\right)$.

Clinical role of miR-133a in HCC analyzed with TCGA data. In order to gain a larger number samples and strengthen the reliability of our results, the authors subsequently searched and downloaded TCGA data for further analysis. Before adding to meta-analysis, they calculated the clinical significance 
A

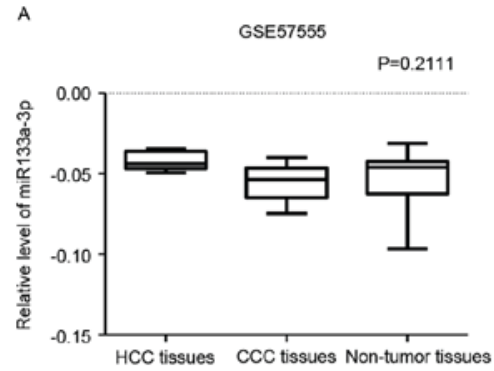

D
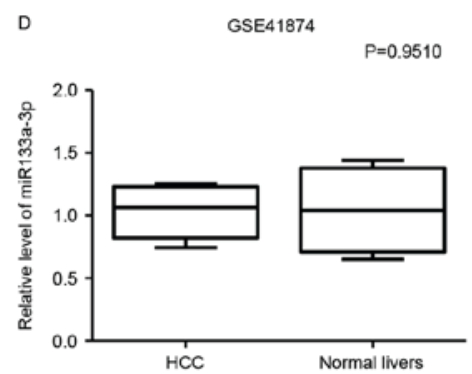

G
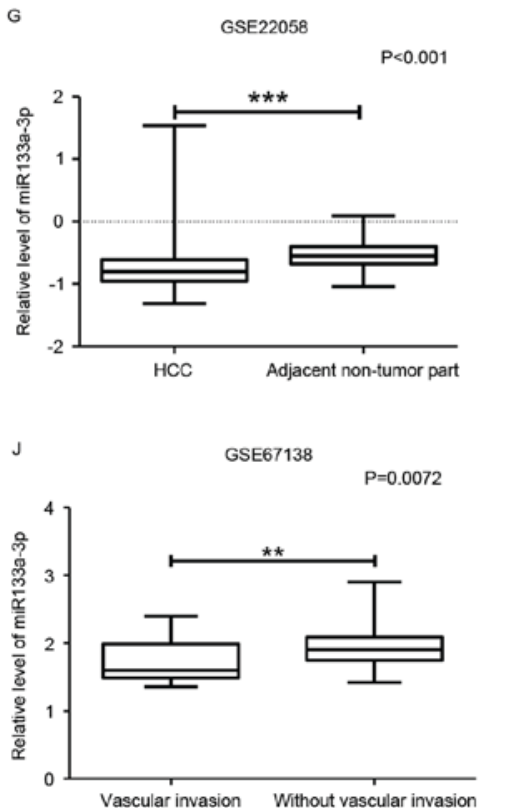

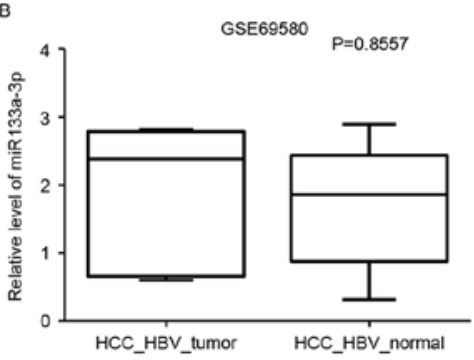

E

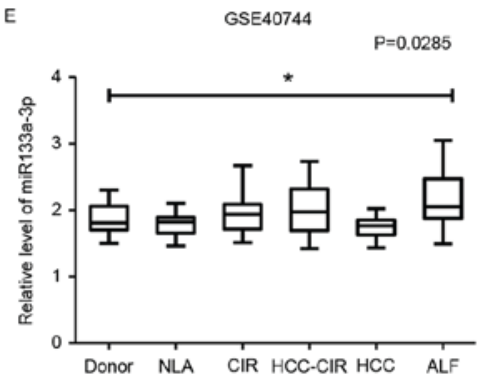

H

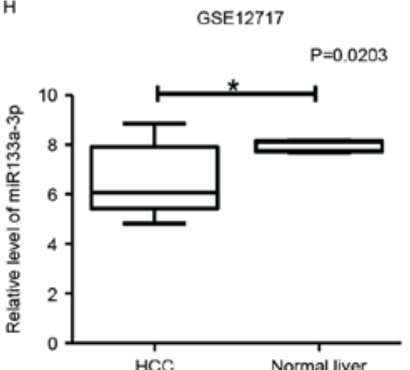

C GSE54751

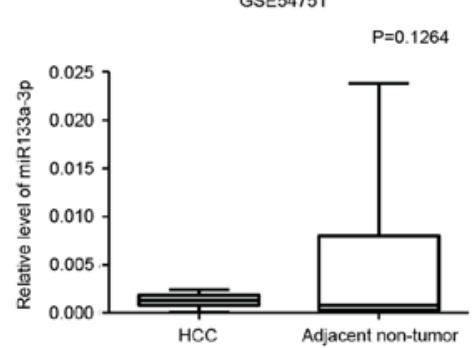

$\mathrm{F}$
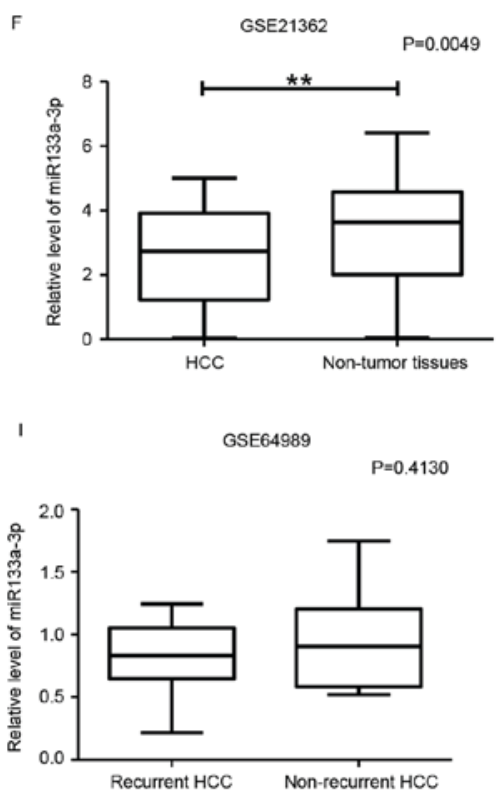

к

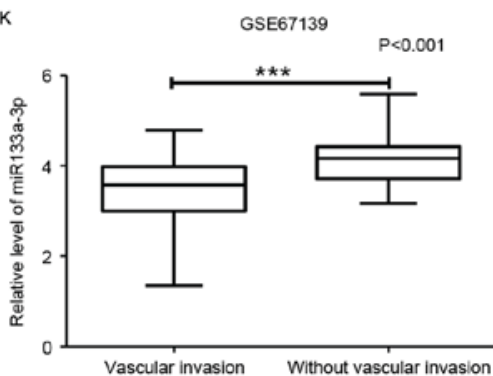

Figure 3. Box-plots presenting miR-133a-3p expression in 11 evaluable microarray chips. (A) miR-133a-3p expression in HCC tissues, CCC tissues and adjacent non-cancerous tissues, $\mathrm{P}=0.2111$. (B) miR-133a-3p expression in HCC tissues and normal liver tissues, $\mathrm{P}=0.8557$. (C) miR-133a-3p expression in HCC and adjacent non-tumor part, $\mathrm{P}=0.1264$. (D) miR-133a-3p expression in primary hepatocellular carcinoma tissues, metastatic hepatocellular carcinoma tissues and normal tissues, $\mathrm{P}=0.9510$. (E) miR-133a-3p expression in HCC tissues, HCC-CIR, CIR, ALF, NLA and none liver cancer donors, $\mathrm{P}=0.0285$. (F) miR-133a-3p expression in HCC tissues and non-tumor tissues, $\mathrm{P}=0.0049$. (G) miR-133a-3p expression in HCC tissues and human healthy liver tissues, $\mathrm{P}<0.001$. (H) miR-133a-3p expression in HCC and normal tissues, $\mathrm{P}=0.0203$. (I) miR-133a-3p expression in recurrent $\mathrm{HCC}$ tissues and non-recurrent $\mathrm{HCC}$ tissues, $\mathrm{P}=0.4130$. (J) miR-133a-3p expression in tumor vascular invasion tissues and tumor vascular invasiveness tissues, $\mathrm{P}=0.0072$. (K) miR-133a-3p expression in tumor vascular invasion tissues and tumor vascular invasiveness tissues, $\mathrm{P}<0.001$. ${ }^{*} \mathrm{P}<0.05,{ }^{* * *} \mathrm{P}<0.01,{ }^{* * * *} \mathrm{P}<0.001$ as indicated. miR, microRNA; $\mathrm{HCC}$, hepatocellular carcinoma; CCC, cholangiocarcinoma; HCC-CIR, HCC surrounding non-tumorous tissue affected by cirrhosis; CIR, HCV-associated cirrhosis without HCC; ALF, HBV-associated acute liver failure; NLA, surrounding normal liver of liver angioma.

of miR-133a-1 in HCC with SPSS 20.0, since no data of the mature miRNA, e.g., miR-133a-3p-1 were available for HCC in TCGA. The results demonstrated that level of miR-133a-1 in $\mathrm{HCC}$ was $3.48 \pm 1.93$, which was reduced markedly when compared to that in non-cancerous liver tissues $(4.44 \pm 1.12$, $\mathrm{P}<0.001$; Fig. 5A). The AUC value of low expression miR-133a-1 for HCC diagnosis was 0.670 ( $\mathrm{P}<0.001$; Fig. 5B). Regarding the difference of miR-133a-1 expression between two corresponding groups of various clinical features, the authors revealed that miR-133a-1 expression in vascular invasion group $(3.32 \pm 1.82)$ demonstrated a significant reduced pattern compared to non-invasiveness group $(3.77 \pm 1.75$, $\mathrm{P}=0.04$; Fig. 5C). The relationship between miR-133a-1 and vascular invasion was confirmed by Spearman correlation test $(\mathrm{r}=-0.127 ; \mathrm{P}=0.029)$. The data of miR-133a-2 was not sufficient for analysis in TCGA database (data not shown).

Meta-analysis with data from GEO, TCGA, PubMed and $P C R$ in house. Since only one publication from the literature search was achieved for the comprehensive meta-analysis, the 


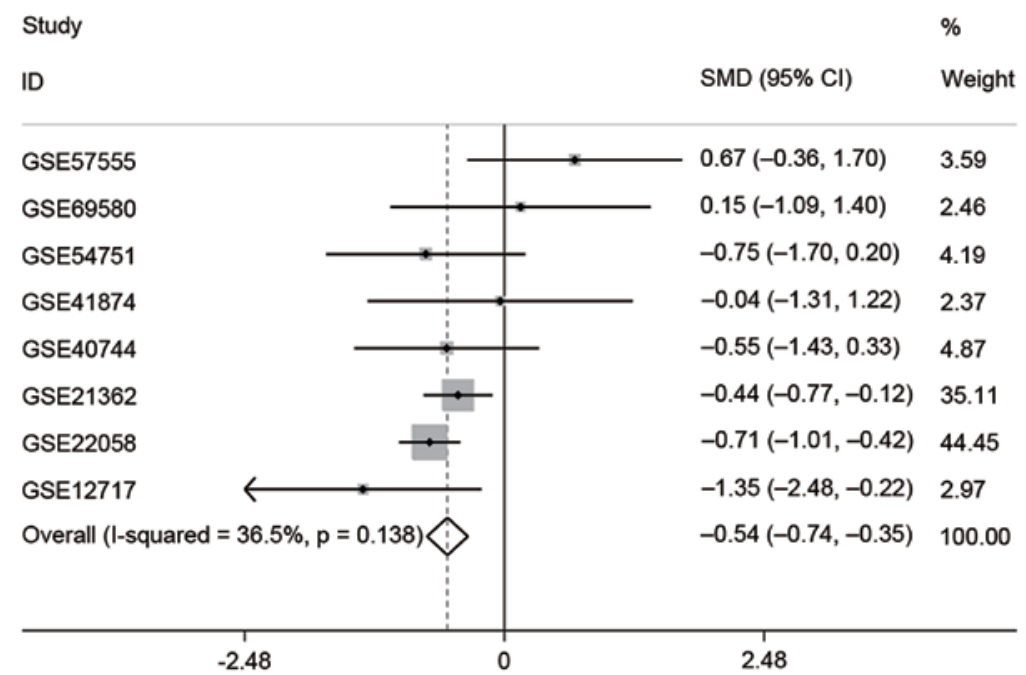

Figure 4. Forest plot of eight microarrays assessing miR-133a-3p expression in HCC. Noticeable difference was observed between tumor and normal groups ( $\mathrm{SMD}=-0.54 ; 95 \% \mathrm{CI},-0.74$ to $-0.35 ; \mathrm{P}<0.001$ ). SMD, standardized mean difference; 95\% CI, 95\% confidence intervals; HCC, hepatocellular carcinoma.

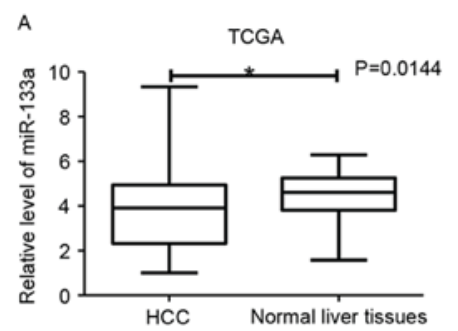

B
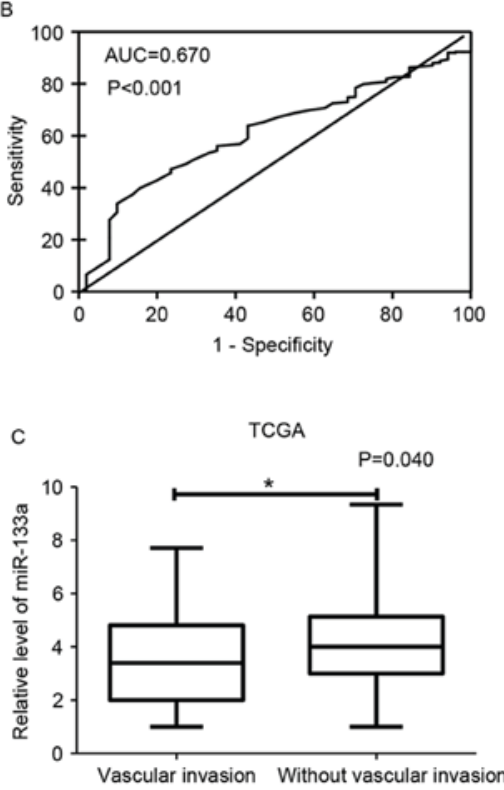

Figure 5. Relative miR-133a-1 expression and its clinical role in HCC with TCGA data. (A) The level of miR-133a-1 in HCC was $3.48 \pm 1.93$, reduced markedly compared to that in non-cancerous liver tissues $(4.44 \pm 1.12$; $\mathrm{P}<0.05)$. (B) The area under the curve value of low expression miR-133a-1 for HCC diagnosis was $0.670(\mathrm{P}<0.001)$. (C) The level of miR-133a-1 in vascular invasion group $(3.32 \pm 1.82)$ revealed a significant reduction compared to invasiveness group $\left(3.77 \pm 1.75 ;{ }^{*} \mathrm{P}<0.05\right.$ as indicated). ${ }^{*} \mathrm{P}<0.05$ as indicated. miR, microRNA; HCC, hepatocellular carcinoma; TCGA, The Cancer Genome Atlas.

data derived from TCGA, PubMed, literature (38) and the PCR data in house based on 95 HCC and matched adjacent normal tissue (data on file) were finally merged in the present meta-analysis. In total, 719 HCC tissues and 404 normal tissues were involved. The number of HCC patients ranged from 5 to 367 per dataset. The involved datasets signatures were showed in Table I. The result generated by fixed-effects model indicated that significant heterogeneity existed among individual datasets $\left(\mathrm{I}^{2}=83.9 \%, \mathrm{P}<0.001\right.$; Fig. 6). Thus, a random-effects model was selected to evaluate the pooled SMD with $95 \% \mathrm{CI}$. The combined SMD suggested that remarkable difference was noted between HCC group and normal control group $(\mathrm{SMD}=-0.69 ; 95 \% \mathrm{CI},-1.10$ to $-0.29 ; \mathrm{P}=0.001$; Fig. 7 ). In other words, miR-133a-3p expression in HCC patients was remarkably lower than that in non-cancerous controls.

Sensitivity analysis. To identify the reason why notable heterogeneity occurred in meta-analysis, the authors conducted a sensitivity analysis (Fig. 6). According to the results, dataset from Zhang et al (38) reported obvious deviation from the estimate. Heterogeneity was found significantly reduced following omission of the dataset of Zhang et al $\left(\mathrm{I}^{2}=18.5 \%\right.$; $\left.\mathrm{P}=0.273\right)$, revealing heterogeneity may be perplexed by the present study. The combined SMD with $95 \%$ CI after removal of data from Zhang et al (SMD=-0.54, 95\% CI, -0.68 to $-0.39, \mathrm{P}<0.001)$ is presented in Fig. 8 .

Publication bias. The authors performed both Begg's funnel plots and Egger's tests for all datasets to evaluate the publication bias. The results generated a Begg's test score of $\mathrm{P}=0.755>0.05$ (Fig. 9) and an Egger's test score of $\mathrm{P}=0.827>0.05$, which suggested the absence of publication bias in the present meta-analysis (Fig. 9).

Targets prediction and functional enrichment. Both DEGs from TCGA and target genes of miR-133a-3p predicted from 12 prediction algorithms were obtained. In total, 4,159 DEGs were retrieved from TCGA. In order to increase the reliability, 3,995 potential target genes were identified by at least five prediction algorithms. Then genes from both approaches (DEGs from TCGA and target genes predicted) were 
Table I. Characteristics of studies included in the meta-analysis.

\begin{tabular}{|c|c|c|c|c|c|c|c|c|c|c|}
\hline \multirow[b]{2}{*}{ Study } & \multirow{2}{*}{$\begin{array}{c}\text { Year } \\
\text { (publication) }\end{array}$} & \multirow[b]{2}{*}{ Country } & \multicolumn{2}{|c|}{ Age (years) } & \multicolumn{2}{|c|}{ Sex } & \multicolumn{2}{|r|}{$\mathrm{n}$} & \multicolumn{2}{|c|}{ Mean \pm standard deviation } \\
\hline & & & $<60$ & $\geq 60$ & Male & Female & $\mathrm{HCC}$ & Normal control & $\mathrm{HCC}$ & Normal \\
\hline GSE57555 & 2015 & Japan & 6 & 15 & 18 & 3 & 5 & 16 & $-0.042 \pm 0.006$ & $-0.053 \pm 0.018$ \\
\hline GSE69580 & 2015 & Taiwan & - & - & - & - & 5 & 5 & $1.854 \pm 1.106$ & $1.697 \pm 0.937$ \\
\hline GSE54751 & 2014 & USA & 6 & 14 & 10 & 10 & 13 & 7 & $0.001 \pm 0.001$ & $0.005 \pm 0.009$ \\
\hline GSE41874 & 2013 & Japan & 9 & 0 & 6 & 3 & 6 & 4 & $1.032 \pm 0.206$ & $1.043 \pm 0.346$ \\
\hline GSE40744 & 2013 & USA & 16 & 5 & 15 & 6 & 9 & 12 & $1.741 \pm 0.171$ & $1.862 \pm 0.247$ \\
\hline GSE21362 & 2011 & Japan & - & - & - & - & 73 & 73 & $2.632 \pm 1.514$ & $3.316 \pm 1.571$ \\
\hline GSE22058 & 2010 & USA & - & - & - & - & 96 & 96 & $-0.746 \pm 0.369$ & $-0.530 \pm 0.216$ \\
\hline GSE12717 & 2008 & USA & 16 & 0 & 4 & 12 & 10 & 6 & $6.475 \pm 1.395$ & $7.997 \pm 0.219$ \\
\hline Zhang et al & 2015 & China & - & - & 24 & 16 & 40 & 40 & $0.510 \pm 0.110$ & $1.050 \pm 0.240$ \\
\hline PCR & 2016 & China & - & - & 75 & 20 & 95 & 95 & $3.285 \pm 2.379$ & $4.497 \pm 2.195$ \\
\hline TCGA & - & - & 172 & 204 & 255 & 122 & 367 & 50 & $3.484 \pm 1.928$ & $4.444 \pm 1.116$ \\
\hline
\end{tabular}

HCC, hepatocellular carcinoma; TCGA, The Cancer Genome Atlas.

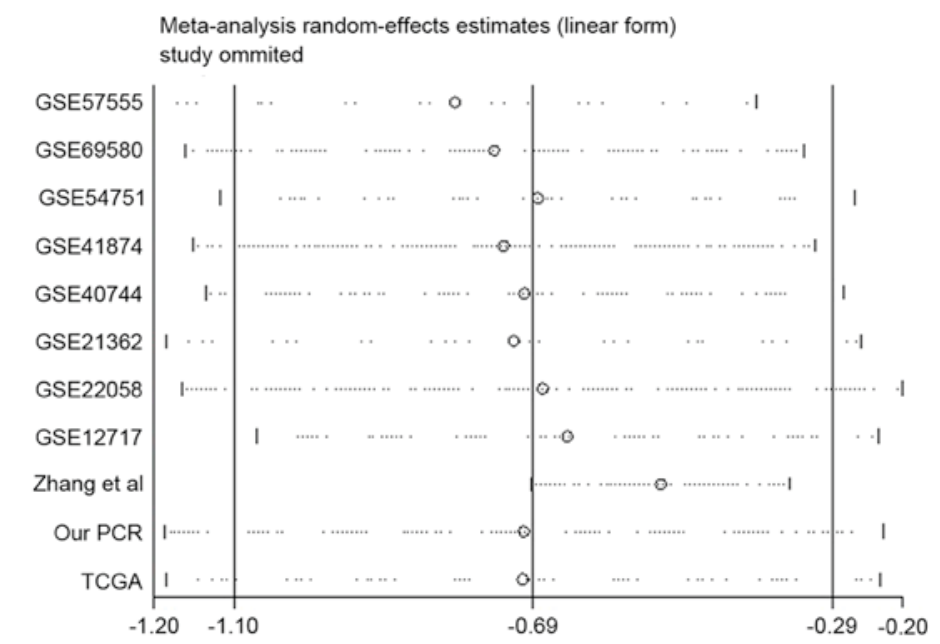

Figure 6. Sensitivity analysis of the 11 evaluable datasets assessing microRNA-133a-3p expression in hepatocellular carcinoma. Significant heterogeneity existed among individual datasets $\left(\mathrm{I}^{2}=83.9 \% ; \mathrm{P}<0.001\right)$, and the dataset from Zhang et al (38) presented an obvious deviation from the estimate.

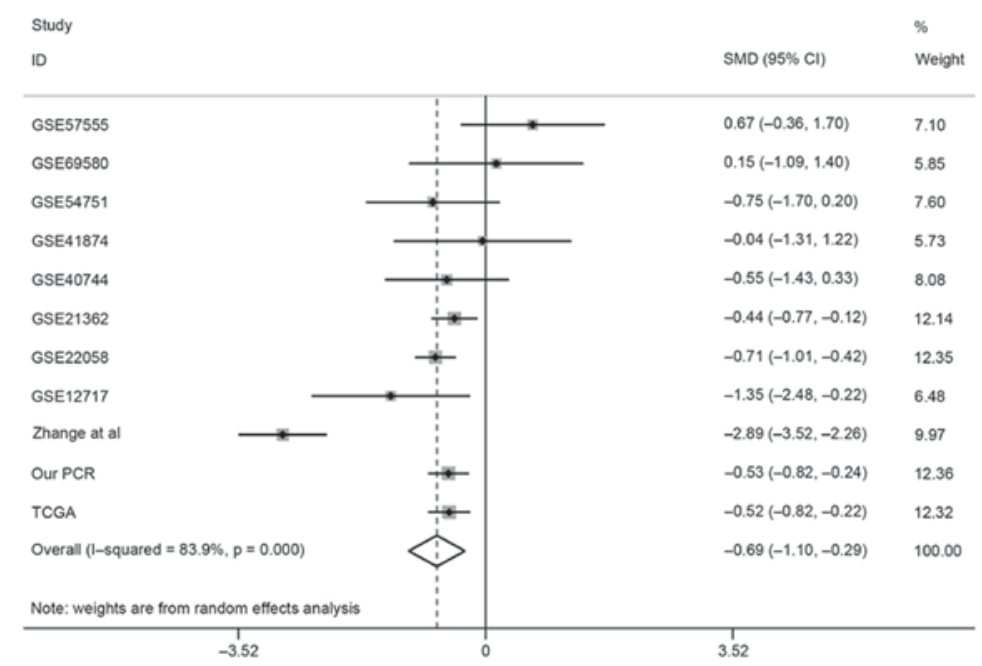

Figure 7. Forest plot of the 11 evaluable datasets assessing microRNA-133a-3p expression in HCC. A random-effects model was selected to evaluate the pooled SMD with 95\% CI. The combined SMD suggested that remarkable difference was noted between HCC group and normal control group (SMD=-0.69; 95\% CI, -1.10 to $-0.29 ; \mathrm{P}=0.001)$. SMD, standardized mean difference; $95 \% \mathrm{CI}, 95 \%$ confidence intervals; HCC, hepatocellular carcinoma. 


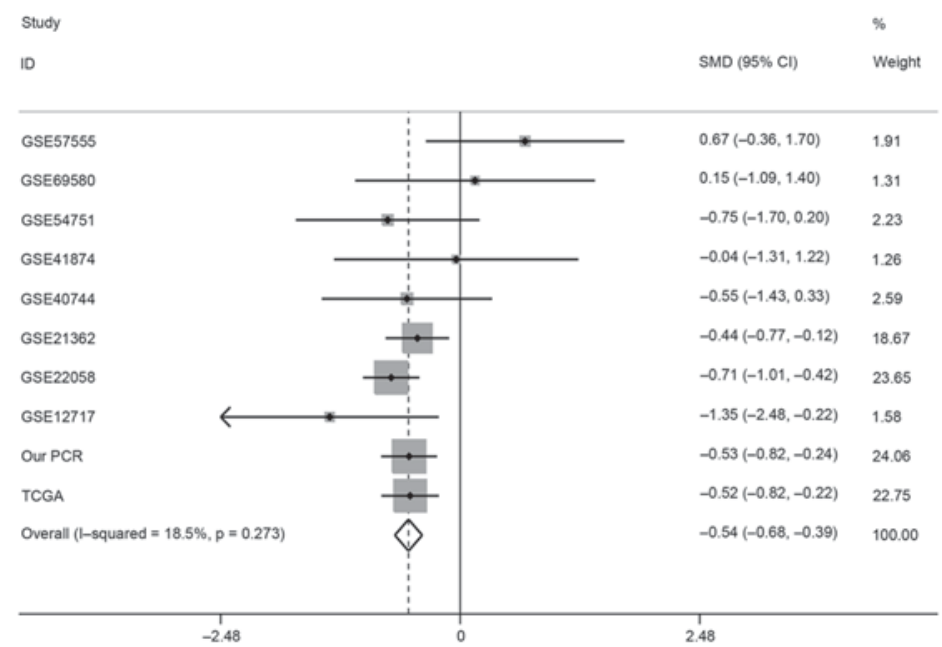

Figure 8. Forest plot of 10 evaluable datasets assessing microRNA-133a-3p expression in hepatocellular carcinoma. The combined SMD with 95\% CI following removal of the data from Zhang et al ( $\mathrm{SMD}=-0.54 ; 95 \% \mathrm{CI},-0.68$ to $-0.39 ; \mathrm{P}<0.001 ; \mathrm{I}^{2}=18.5 \% ; \mathrm{P}=0.273$ ) presented a noticeable difference between tumor and normal groups. SMD, standardized mean difference; $95 \%$ CI, $95 \%$ confidence intervals.

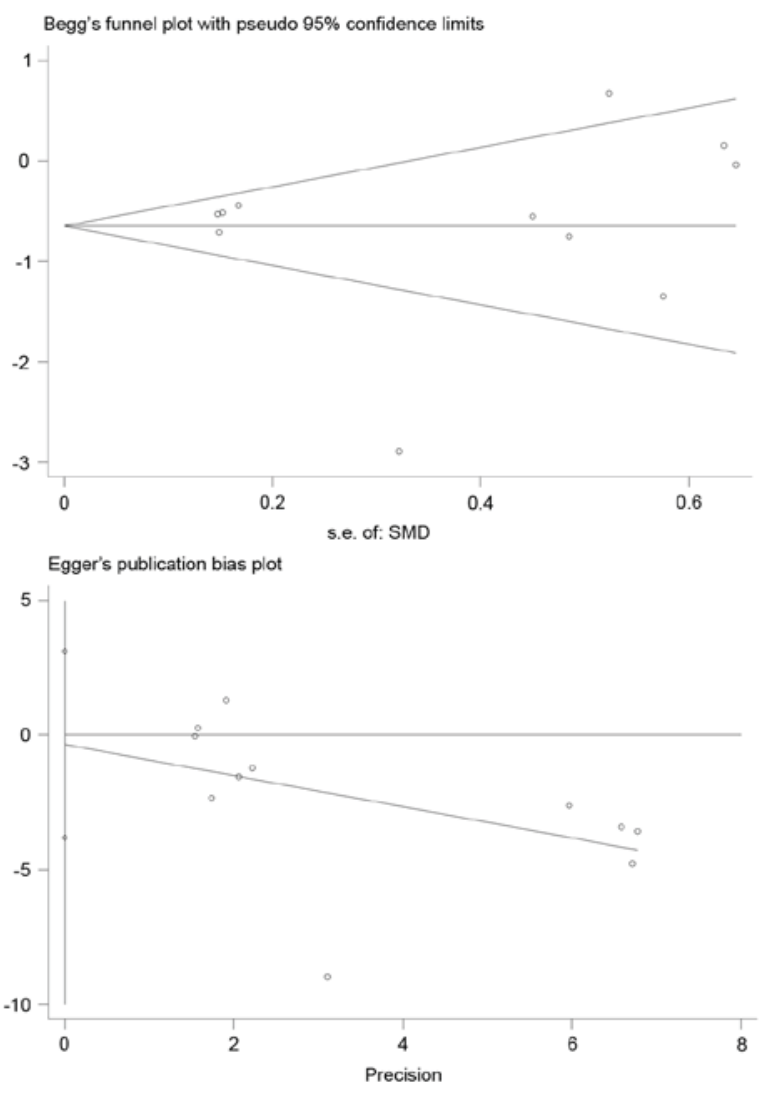

Figure 9. Funnel blot and Egger's plot for the publication bias test of 11 studies. SMD, standardized mean difference.

overlapped and 828 putative targets were selected for further functional analyses.

In the three categories of GO pathway analyses, target genes were statistically enriched in biological process (BP), in which 372 terms were identified and FDR were $<0.05$ in 21 terms. The top enriched BP term was cell-cell signaling (GO: 0007267), which contained 70 genes, and the FDR reached $4.02 \times 10^{-9}$. Besides, the top term of cellular components (CC) and molecular functions (MF) were the plasma

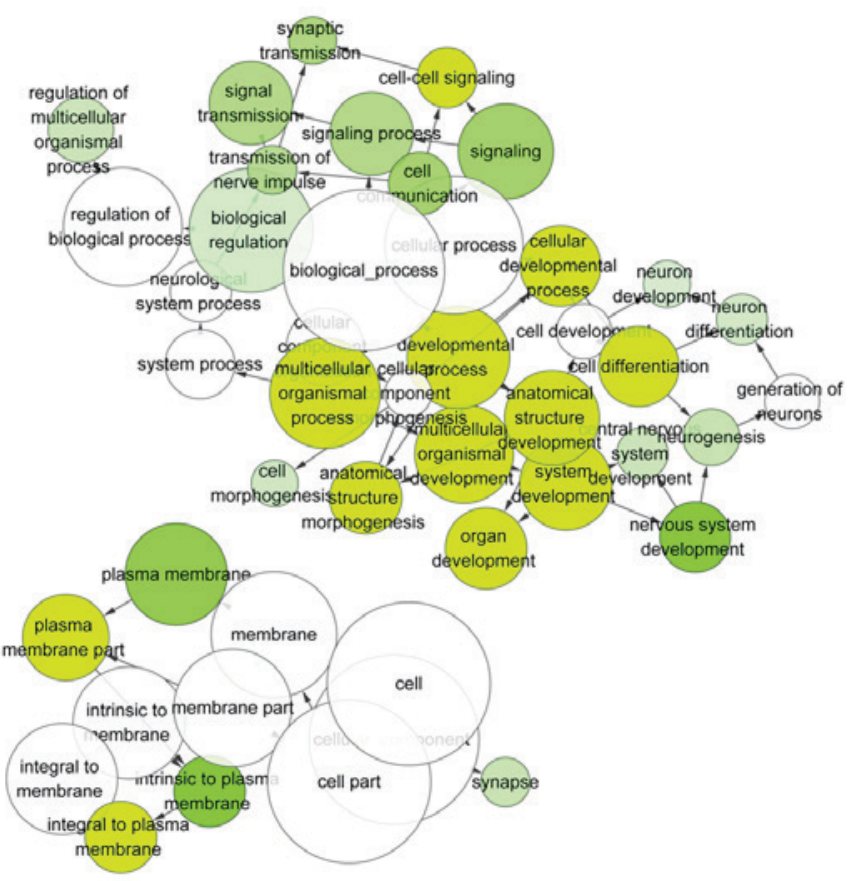

Figure 10. Gene Ontology pathway interaction maps were drawn using the Cytoscape v3.4.0 BiNGO plug-in. Color and size intensity denotes the significance of functional pathway and the enrichment of gene, dark color represents the most significant pathway terms, and the larger size represents more enrichment of genes.

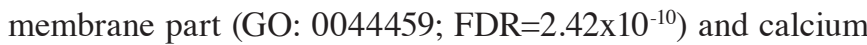
ion binding (GO: 0005509; FDR=0.014), respectively. The top 10 terms of each category are listed in Table II, and the GO pathway map is presented in Fig. 10.

The KEGG pathways were also identified, many of which were related to signaling pathway, such as the calcium signaling pathway (hsa04020; $\mathrm{P}=2.94 \times 10^{-4}$ ), neuroactive ligand-receptor interaction (hsa04080; $\mathrm{P}=0.001$ ) and the Hedgehog signaling pathway (hsa04340; $\mathrm{P}=0.005$ ). The target genes were significantly enriched in PANTHER terms of Synaptic-vesicle-trafficking ( $\left.\mathrm{P} 05734 ; \mathrm{P}=4.32 \times 10^{-5}\right)$, the 


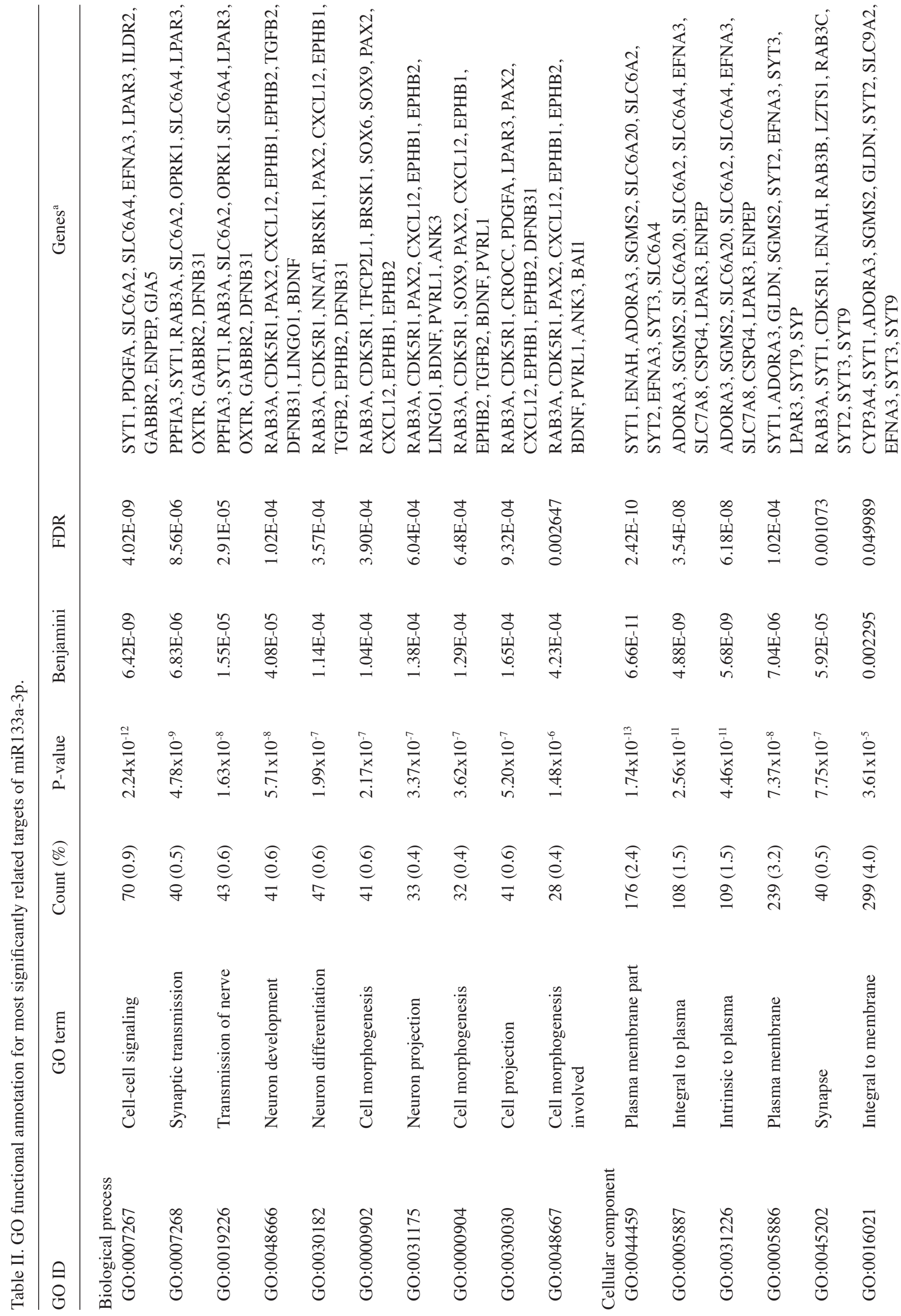




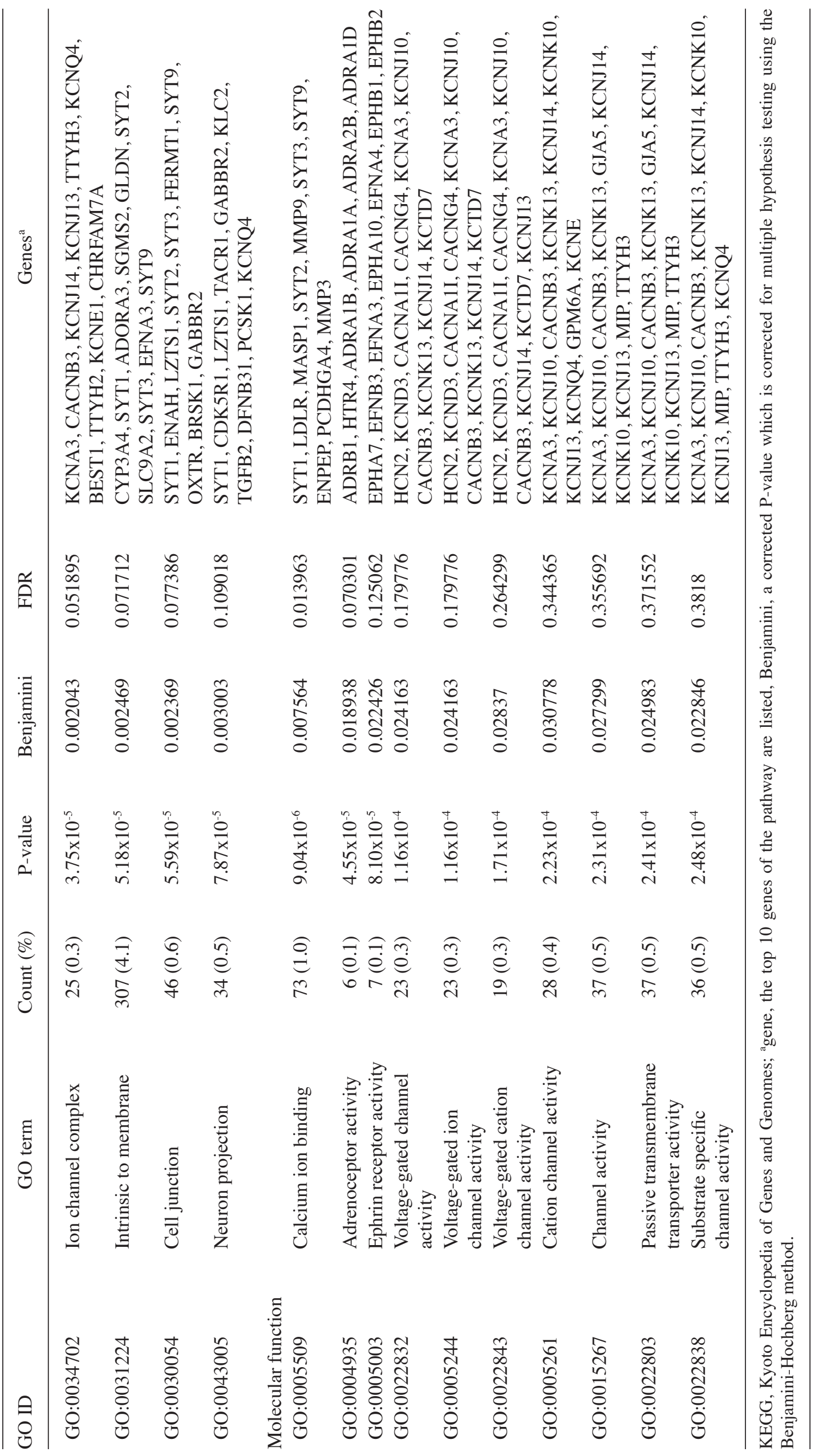




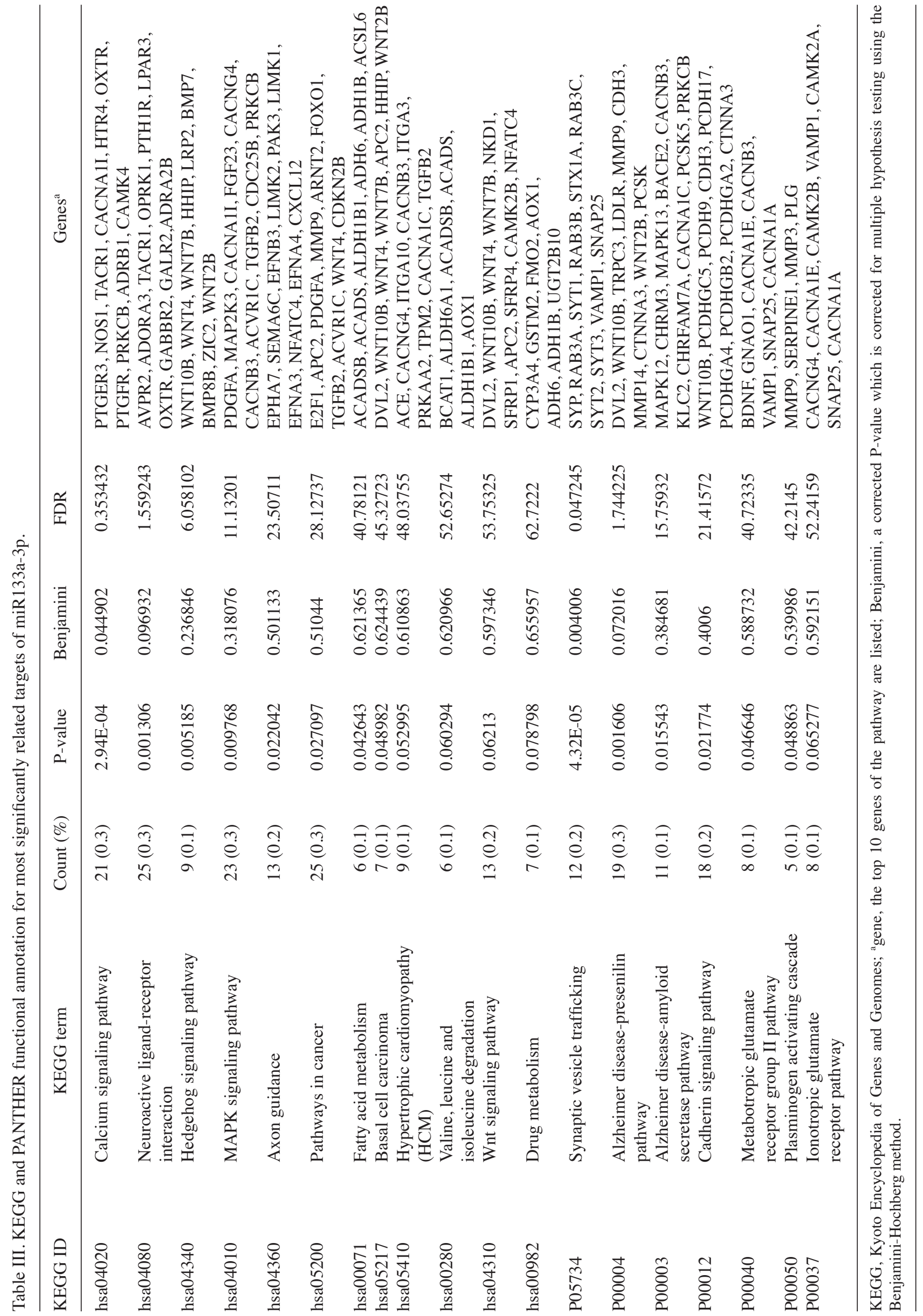




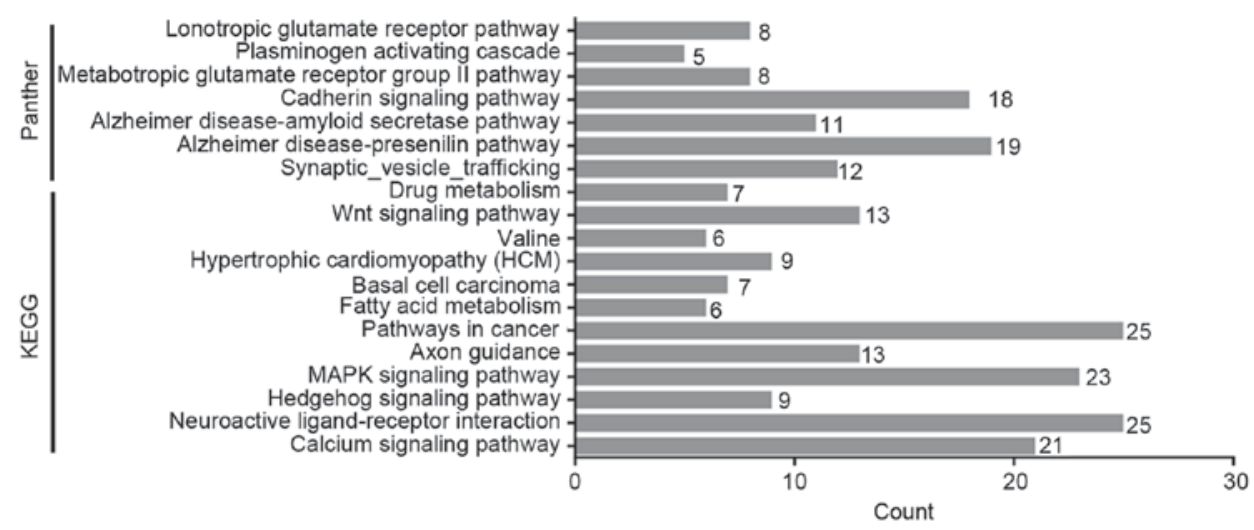

Figure 11. Significantly enriched annotation of KEGG and PANTHER pathway of the potential targeted by miR-133a-3p in hepatocellular carcinoma. KEGG, Kyoto Encyclopedia of Genes and Genomes; MAPK, mitogen-associated protein kinase.

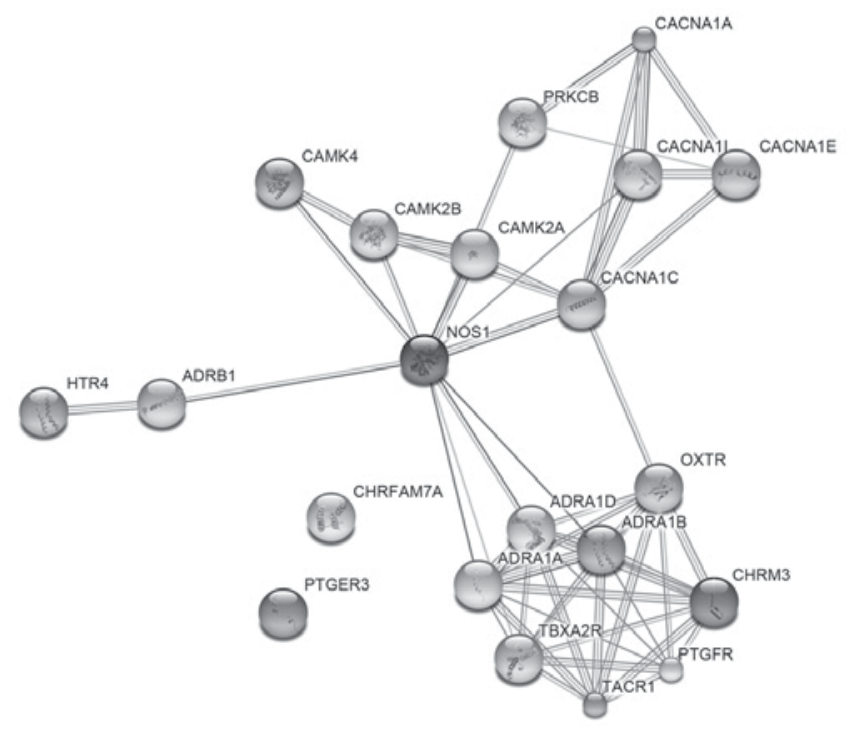

Figure 12. Protein-protein interaction networks of genes in the calcium signaling pathway. Interactions among 21 genes were illustrated using the STRING online database (http://string-db.org). Network nodes represent proteins, and edges represent protein-protein associations.

Alzheimer disease-presenilin pathway $(\mathrm{P} 00004, \mathrm{P}=0.002)$, the Alzheimer disease-amyloid secretase pathway (P00003; $\mathrm{P}=0.016$ ). All KEGG and PANTHER pathway terms are listed in Table III and Fig. 11.

PPIs network of calcium signaling pathway. Proteins rarely act alone as their functions tend to be regulated. PPIs refer to lasting or ephemeral physical contacts of high specificity which was established between two or more protein molecules as a result of biochemical events steered by electrostatic forces including the hydrophobic effect. Commonly they refer to physical contacts with molecular associations between chains that occur in a cell or in a living organism in a specific biomolecular context. Thus, the authors decided to further analyze the most significant pathway of KEGG by constructing PPIs network. So 'Calcium signaling pathway' with 21 genes was included for the PPI analysis. As a result, a total of five key genes with the number of more than eight edges indicating higher connectivity degree were identified, including NOS1,
ADRA1A, ADRA1B, ADRA1D and TBXA2R. PPIs network were presented in Fig. 12.

Validation of some potential target gene expression based on TCGA dataset. Among all the significant pathways in the present study, the Calcium signaling pathway (KEGG ID: hsa04020) was selected for validation. There were 21 genes involved in the pathway. The authors then analyzed the relative expression (on a $\log 2$ scale) of these 21 predicted genes of miR-133a-3p by using TCGA HCC dataset. Eight genes (NOS1, PRKCB, ADRA1A, ADRB1, TBXA2R, CAMK4, ADRA1B, CAMK2B) were downregulated and they could not be real targets of miR-133a-3p due to same trend of the expression in HCC (Table IV). It was interesting that 11 among the rest 13 genes presenting extremely significant overexpressed pattern in liver cancers as compared to that in the adjacent liver tissues ( $\mathrm{P}<0.01$; Table IV; Fig. 13A-K), such as CACNA1E, HTR4, CACNA1C, CHRM3, OXTR, CACNA1I, CACNA1A, CHRFAM7A, PTGER3, TACR1, CAMK2A.

\section{Discussion}

As the investigation surrounding miRNA function is currently very significant in research, more and more miRNAs have been reported to exert functions in HCC and development (45-54). Downregulated miR-133a-3p has been identified in several types of cancers, such as bladder (55), colorectal (15), osteosarcoma (56-58), non-small-cell lung $(36,59)$ and esophageal cancers (60). However, the expression of miR-133a-3p in HCC tissues remains controversial. Most pilot studies proposed that miR-133a-3p may play as a tumor suppressor in HCC, while several studies present inconsistency (GSE57555, GSE69580). How miR-133a-3p exerts its function is still unclear. Comparing the data collected from GEO datasets, TCGA and literature, the authors found that the uneven sample size from different studies may contribute to the contradictory results. In this meta-analysis, miR-133a-3p expression was pooled from eight microarray datasets from GEO and the other three studies from literature, TCGA and the authors' own PCR data. The result generated by fixed-effects model indicated that significant heterogeneity was present among individual datasets $\left(I^{2}=83.9 \%\right.$; $P<0.001$; Fig. 6$)$. The significant heterogeneity may result from the following reasons: Firstly, different 
Table IV. Expressions of 21 genes based on The Cancer Genome Atlas dataset (374 HCC tissues and 50 normal liver tissues).

\begin{tabular}{|c|c|c|c|c|c|}
\hline Gene name & $\begin{array}{l}\text { HCC tissue } \\
(\text { Mean } \pm \mathrm{SD})\end{array}$ & $\begin{array}{l}\text { Normal liver tissue } \\
\quad(\text { Mean } \pm \text { SD })\end{array}$ & $\mathrm{t}$ & P-value & $\mathrm{FC}$ \\
\hline CACNA1E & $3.86 \pm 2.16$ & $1.81 \pm 1.33$ & -5.97 & $3.64 \times 10^{-6 a}$ & 2.05 \\
\hline HTR4 & $3.52 \pm 1.89$ & $2.13 \pm 1.14$ & -5.82 & $5.36 \times 10^{-7 a}$ & 1.4 \\
\hline CACNA1C & $6.5 \pm 1.65$ & $5.38 \pm 1.03$ & -6.62 & $2.81 \times 10^{-9 a}$ & 1.12 \\
\hline CHRM3 & $6.3 \pm 2.12$ & $5.23 \pm 1.4$ & -4.7 & $1.02 \times 10^{-5 a}$ & 1.07 \\
\hline OXTR & $4.91 \pm 1.79$ & $3.86 \pm 1.66$ & -3.83 & $1.47 \times 10^{-4 a}$ & 1.04 \\
\hline CACNA1I & $3.51 \pm 2.18$ & $2.51 \pm 1.24$ & -4.03 & $1.60 \times 10^{-4 a}$ & 1.00 \\
\hline CACNA1A & $3.73 \pm 1.3$ & $2.85 \pm 0.82$ & -6.44 & $7.71 \times 10^{-9 a}$ & 0.88 \\
\hline CHRFAM7A & $2.25 \pm 1.35$ & $1.38 \pm 0.48$ & -4.22 & $7.54 \times 10^{-4 a}$ & 0.86 \\
\hline PTGER3 & $4.88 \pm 1.73$ & $4.03 \pm 1.42$ & -3.23 & $1.35 \times 10^{-3 a}$ & 0.84 \\
\hline TACR1 & $3.21 \pm 1.7$ & $2.38 \pm 0.91$ & -4.65 & $1.46 \times 10^{-5 a}$ & 0.84 \\
\hline CAMK2A & $3.6 \pm 2.19$ & $2.82 \pm 1.03$ & -3.94 & $1.34 \times 10^{-4 a}$ & 0.78 \\
\hline ADRA1D & $3.61 \pm 2.26$ & $3.27 \pm 1.74$ & -0.72 & $4.69 \times 10^{-1}$ & 0.34 \\
\hline PTGFR & $5.92 \pm 3.04$ & $5.6 \pm 1.21$ & -1.37 & $1.74 \times 10^{-1}$ & 0.32 \\
\hline NOS1 & $2.64 \pm 1.84$ & $2.97 \pm 0.98$ & 1.66 & $9.98 \times 10^{-2}$ & -0.33 \\
\hline PRKCB & $6.67 \pm 1.65$ & $7.78 \pm 1.43$ & 4.56 & $6.62 \times 10^{-6 a}$ & -1.12 \\
\hline ADRA1A & $5.78 \pm 2.62$ & $6.95 \pm 4.59$ & 1.76 & $8.35 \times 10^{-2}$ & -1.17 \\
\hline ADRB 1 & $3.71 \pm 1.76$ & $5.03 \pm 1.49$ & 5.71 & $2.37 \times 10^{-7 a}$ & -1.33 \\
\hline TBXA2R & $6.87 \pm 1.09$ & $8.32 \pm 1.17$ & 8.28 & $1.46 \times 10^{-11 a}$ & -1.45 \\
\hline CAMK4 & $5.25 \pm 1.44$ & $7 \pm 1.09$ & 10.17 & $1.09 \times 10^{-15 a}$ & -1.74 \\
\hline ADRA1B & $6.55 \pm 1.86$ & $8.47 \pm 0.74$ & 13.47 & $8.19 \times 10^{-28 a}$ & -1.92 \\
\hline CAMK2B & $5.32 \pm 2.63$ & $8.55 \pm 0.89$ & 17.13 & $3.33 \times 10^{-41 \mathrm{a}}$ & -3.23 \\
\hline
\end{tabular}

FC, fold change; SD, standard deviation; HCC, hepatocellular carcinoma. ${ }^{a} \mathrm{P}<0.001$ vs. normal tissue.

laboratories differed broadly as a result of the inter-platform alterations. The eight included GEO datasets utilized various platforms of microarray by different research teams; thus, there existed significant heterogeneity. Secondly, the samples were from different countries, which indicated that different races may lead to the significant heterogeneity. The eight GEO datasets included four datasets from USA (GSE40744, GSE54751, GSE22058 and GSE12717), three datasets from Japan (GSE57555, GSE41874 and GSE21362) and one dataset (GSE69580) from Taiwan, while data of the literature and the authors' own PCR were from mainland China. In addition, two ethnicities, Asian and Caucasian, were pooled in this meta-analysis. Furthermore, different techniques to detect the expression of miR-133a-3p may also result in the significant heterogeneity. In the present meta-analysis, the eight microarray datasets from GEO, the studies from literature and the authors' own PCR data used different means to detect the expression of miR-133a-3p. The studies from the literature and our own data both used PCR to detect the expression of miR-133a-3p, while the eight GEO datasets used difference microarray/RNA-seq platforms. When the data from Zhang et al (38) was excluded, the heterogeneity changed from 83.9 to $18.5 \%$, which suggested that heterogeneity may be perplexed by this dataset. However, their result indicated that miR-133a-3p could function as a tumor suppressor in $\mathrm{HCC}$, which was consistent with our combined effect of miR-133a-3p. A total of 40 pairs of HCC tissue samples of the study of Zhang et al (38) were collected from the First Hospital of Jilin University (Changchun, China).
Compared to other case-control studies, the authors hypothesize that the limited sample sizes could be the main reason for the current heterogeneity. Wang et al (61) investigated 10 primary HCC samples as well as their matched normal adjacent hepatic tissues, and demonstrated that miR-133a-3p level was reduced in HCC. However, this study was not included into this meta-analysis, for the means and standard deviation or original data could not be collected. Furthermore, Wang et al (61) also stated that overexpression of miR-133a-3p suppressed cell growth, migration and invasion, whereas the overexpression of miR-133a-3p enhanced apoptosis in HCC cells. Though, some evidence reported that miR-133a-3p level was reduced in HCC tissue, the non-invasive detection is more valuable for clinic; therefore, the miR-133a-3p level in the serum of HCC patients presents more clinical practice significance. Lin et al (62) compared 108 patients with HCC and 149 matched controls also indicated that miR-133a notably expressed in the serum of HCC. In their study, the authors identified a miRNA classifier $\left(\mathrm{C}_{\mathrm{mi}}\right)$ containing seven differentially expressed miRNAs (miR-29a, miR-29c, miR-133a, miR-143, miR-145, miR-192, and miR-505) was a potential biomarker for HCC. It could identify small-size, early-stage and $\alpha$-fetoprotein-negative $\mathrm{HCC}$ in patients at risk. The diagnosis value of seven-miRNA combination achieved high performance (AUC>0.800 and accuracy $>80 \%$ ). However, Lin et al (62) indicated that miR-133a level in the serum of HCC increased when compared with the control patients with chronic hepatitis $\mathrm{B}$, which was inconsistent with the authors' 

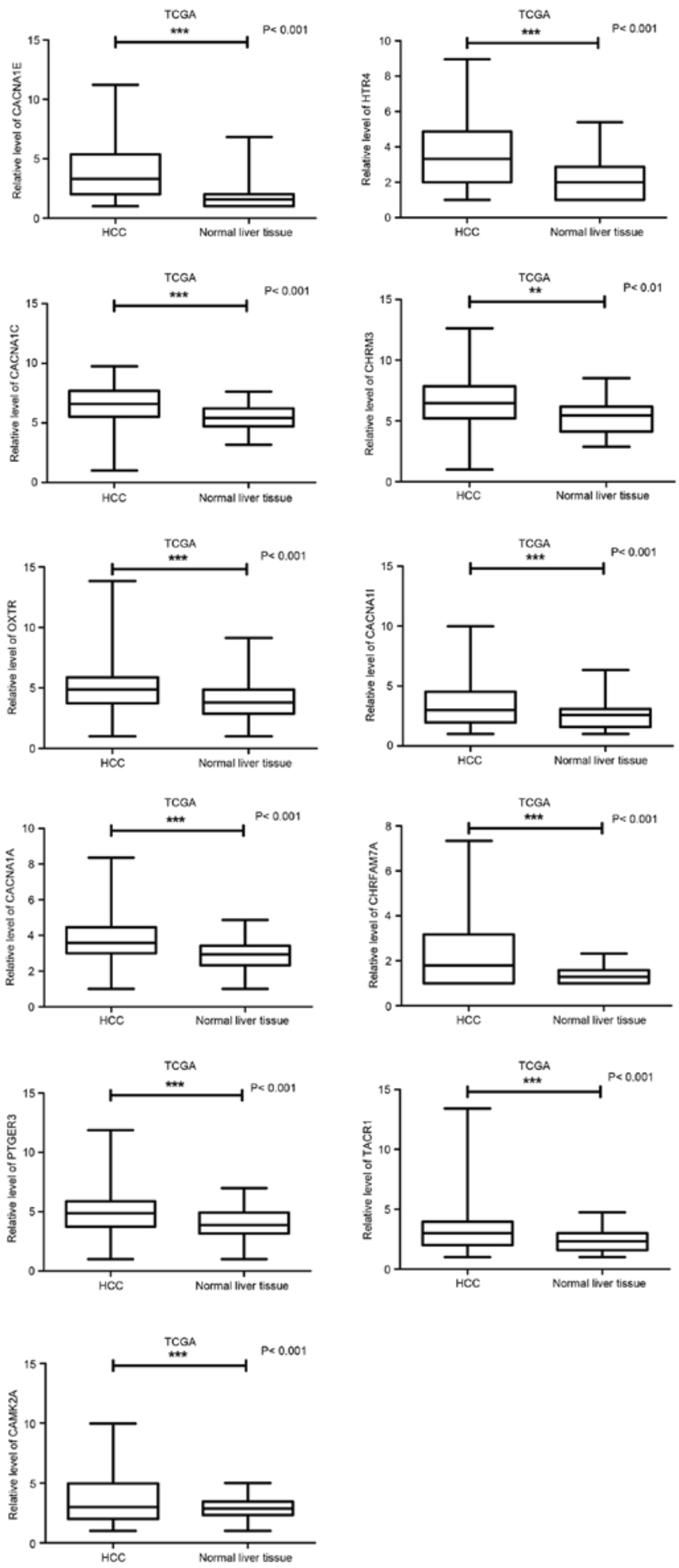

Figure 13. Expressions of the 11 genes with significant fold changes from the calcium signaling pathway in HCC based on TCGA dataset. The TCGA RNAseq profiles of $\mathrm{HCC}$ were extracted to explore the relative expressions of 11 selected genes from the calcium signaling pathway in HCC tissue and normal liver tissue (on a $\log 2$ scale), ${ }^{* *} \mathrm{P}<0.01,{ }^{* * *} \mathrm{P}<0.001$ as indicated. CACNA1E; HTR4; CACNA1C; CHRM3; OXTR; CACNA1I; CACNA1A; CHRFAM7A; PTGER3; TACR1; CAMK2A. HCC, hepatocellular carcinoma; TCGA, The Cancer Genome Atlas.

present result. The different expression trend of miR-133a-3p between tissue and serum might because of the different patient samples and detection methods. For example, the matched controls of this study included 51 healthy controls, 51 patients with chronic hepatitis B, and 47 with $\mathrm{HBV}$-induced liver cirrhosis; miRNAs level were detected by reverse transcription-quantitative PCR. Thus, more explorations are required to fig. out the miR-133a-3p level in the serum of HCC patients.

Even though eight microarray gene sets did not show noticeable heterogeneity in the meta-analysis, two of them (GSE57555 and GSE69580) reported that miR-133a-3p expression was higher in HCC than in normal tissues. One (GSE41874) demonstrated no significant difference between HCC and normal tissues. Having analyzed the three microarrays (GSE57555, GSE69580 and GSE41874), the authors noticed that the number of normal samples $(n=16)$ was obviously larger than that of HCC tissues $(n=5)$ in GSE57555, while both tumor and normal samples were small in GSE69580 ( $n=5$ for HCC, $n=5$ for control) and GSE41874 ( $n=6$ for HCC, $n=4$ for control). It should be noted that, CIs intervals showed the same trend, which provides more reliable evidence that miR-133a-3p serves a suppressive role in HCC. Furthermore, miR-133a-3p could also be a diagnostic marker for HCC.

In the present study, the authors observed that miR-133a-1 expression in vascular invasion group $(3.32 \pm 1.82)$ revealed a significantly reduced pattern compared to the non-invasiveness group ( $3.77 \pm 1.75 ; \mathrm{P}=0.04$; Fig. 5) from the TCGA data. Similarly, in one GEO dataset (GSE67138), the expression of miR-133a-3p in the vascular invasion group also showed significant reduced pattern compared to without vascular invasion group ( $\mathrm{P}=0.0072$; Fig. $3 \mathrm{~J})$. It was indicated that decreased expression of miR-133a-3p was closely correlated with vascular invasion. Meanwhile, the present functional analyses indicated that several target genes of miR-133a-3p were both involved in the calcium signaling pathway and the mitogen-associated protein kinase (MAPK) signaling pathway; from which the calcium signaling pathway may involve in the vascular invasion of HCC (63), and the MAPK signaling pathway also has been reported to promote cell growth and invasion in HCC (64). Thus, miR-133a-3p may exert its effect of vascular invasion by targeting CACNA1A, CACNA1C, CACNA1E, CACNA1I and PRKCB in the calcium signaling pathway and the MAPK signaling pathway.

The mature miRNA microRNA-133a-3p (miR-133a-3p, previously named miR-133a) can be generated from miR-133a-1 and miR-133a-2 (the precursor-miRNA, pre-miRNA). miR-133a-1 and miR-133a-2 are located on different chromosomes and have different sequences, with diverse functions in the process of transcription (65). Theoretically, miRNAs are transcribed as long primary-miRNA (pri-miRNA) transcripts that are cleaved in the nucleus by the DROSHA (Drosha) enzyme to liberate the precursor-miRNA (pre-miRNA) hairpin. The pre-miRNA is subsequently exported from the nucleus and further processed by the enzyme DICER1 (Dicer) in the cytoplasm to produce mature miRNAs. miR-133a is encoded by two separate genes in humans (miR-133a-1 and miR-133a-2). miR-133a-1 is located on chromosome 18 (MI0000450), and miR-133a-2 is located on chromosome 20 (MI0000451). The processing of the precursor transcripts by Dicer generates mature miRNAs: miR-133a-3p. 
The different sequences of miR-133a-1, miR-133a-2 and miR-133a-3p are as follows:

>hsa-mir-133a-1 MI0000450

ACAAUGCUUUGCUAGAGCUGGUAAAAUGGAACC AAAUCGCCUCUUCAAUGGAUUUGGUCCCCUUCA ACCAGCUGUAGCUAUGCAUUGA

\section{>hsa-mir-133a-2 MI0000451 \\ GGGAGCCAAAUGCUUUGCUAGAGCUGGUAAAAU GGAACCAAAUCGACUGUCCAAUGGAUUUGGUCC CCUUCAACCAGCUGUAGCUGUGCAUUGAUGGCG CCG}

\section{>hsa-miR-133a-3p MIMAT0000427 UUUGGUCCCCUUCAACCAGCUG}

miR-133a-1, miR-133a-2 and miR-133a-3p have different sequences and different targets bind to their promoter region in the transcription or translation process. Based on the understanding of their diverse functions, the authors intend to understand disease progression more precisely, and this may provide new insights for disease treatment.

The molecular mechanism of miR-133a-3p in HCC is of great importance to understand the carcinogenesis of HCC. Previous studies have reported that miR-133a-3p may target fascin actin-bundling protein 1 (FSCN1), insulin-like growth factor 1 receptor (IGF-1R), epidermal growth factor receptor, transgelin 2, matrix metallopeptidase-9 (MMP-9), ATPase copper transporting $\beta$, death associated protein kinase 2 , SRY-box 4 and serum response factor, to exert its function in different kinds of tumors. Furthermore, FSCN1, IGF-1R and MMP-9 have been described as targets of miR-133a-3p and influenced cell proliferation, colony formation, migration and invasion in HCC cells. Thus, the authors deduce that miR-133a-3p may target multiple mRNAs in different signaling pathways of HCC. To get the whole picture of miR-133a-39 related potential target networks, they conducted targets prediction and functional pathway analyses. A total of 12 target prediction tools were used for collection of the potential targets genes. In order to minimize false positives, the potential target genes need to be predicted in at least five in silico tools out of 12 . Subsequently, predicted targets were overlapped with DEGs from TCGA, which are representative of key genes in the tumorigenesis of HCC, to eventually obtain final target genes of miR-133a-3p in HCC. Then functional analysis was conducted to investigate potential molecular pathway of miR-133a-3p, which will contribute to the understanding of molecular mechanisms of the tumorigenesis and progression of HCC. The possible multiple pathways suggested that miR-133a-3p can potentially regulate many of the required steps in HCC development, from variations in cell morphogenesis to channel activity, as well as various signaling pathways. The molecular functions of these pathways may initiate a new trial on HCC pathogenesis. These pathways could be a prospective source of novel treatment targets and markers. Five key genes NOS1, ADRA1A, ADRA1B, ADRA1D and TBXA2R were obtained from PPIs network of the calcium signaling pathway, which was the most significantly enriched pathway achieved from KEGG, are potentially targeted by
miR-133a-3p in HCC. However, these five hub genes have not yet been researched in HCC; thus further experimental validation is needed.

Some limitations should be noted in the present study. Firstly, significant heterogeneity was observed when all data were pooled together in the meta-analysis. Subgroup analyses have not been designed due to the small number of included studies. Other confounding introduced by RNA extraction or individual background may also disturb the result of the meta-analysis. Secondly, the small sample size limited the robustness of the meta-analysis conclusion. Further studies entailing large sample size should be designed to confirm miR-133a-3p expression levels in HCC. Thirdly, the potential target genes and pathways were only predicted by bioinformatics without experimental verification. The authors' group plans to perform in vitro and in vivo experiments to validate these in silico findings in the future.

To better explore the possibility of the target candidates from simple prediction, the authors further inspected the gene expression data from TCGA dataset and confirmed the overexpression of 13 of 21 genes described in the calcium signaling pathway (KEGG ID: hsa04020), which was the most significant KEGG term. Especially, six genes were dramatically upregulated in liver tumor tissues, giving an FC of $>1$. These genes may have more possibility to be the real targets of miR-133a-3p in HCC due to their noteworthy overexpression in cancer tissues. Of course, the results need to be further confirmed with other experiments.

The present study mined the publicly available data, including GEO, TCGA, published study and the authors' own PCR data together to study the possible clinical role of miR-133a-3p. The results suggested that miR-133a-3p may play as a tumor suppressor in HCC. Furthermore, the prospective novel pathways of miR-133a-3p could offer potential biomarkers for HCC. To the best of the authors' knowledge, this is the first study to combine multiple resources to validate miR-133a-3p suppressive role in HCC and to explore its molecular mechanism by functional pathway analysis. However, the findings need further experimental confirmation.

\section{Acknowledgements}

This study was supported partly by the Fund of Guangxi Medical University Students Innovative Project (grant no. 201610598092).

\section{References}

1. Siegel RL, Miller KD and Jemal A: Cancer statistics, 2015. CA Cancer J Clin 65: 5-29, 2015.

2. Yu G, Chen X, Chen S, Ye W, Hou K and Liang M: Arsenic trioxide reduces chemo-resistance to 5-fluorouracil and cisplatin in HBx-HepG2 cells via complex mechanisms. Cancer Cell Int 15: 116, 2015.

3. Bao J, Lu Y, Deng Y, Rong C, Liu Y, Huang X, Song L, Li S and Qin X: Association between IL-18 polymorphisms, serum levels, and HBV-related hepatocellular carcinoma in a Chinese population: A retrospective case-control study. Cancer Cell Int 15: 72, 2015.

4. Luo Y, Zhang X, Tan Z, Wu P, Xiang X, Dang Y and Chen G: Astrocyte elevated gene-1 as a novel clinicopathological and prognostic biomarker for gastrointestinal cancers: A meta-analysis with 2999 patients. PLoS One 10: e0145659, 2015. 
5. Yu MA, Liang P, Yu XL, Han ZY, Dong XJ, Wang YU, Cheng C and Li X: Multiple courses of immunotherapy with different immune cell types for patients with hepatocellular carcinoma after microwave ablation. Exp Ther Med 10: 1460-1466, 2015.

6. Luo X, Yang S, Zhou C, Pan F, Li Q and Ma S: MicroRNA-328 enhances cellular motility through posttranscriptional regulation of PTPRJ in human hepatocellular carcinoma. Onco Targets Ther 8: 3159-3167, 2015

7. Li T, Zhao S, Song B, Wei Z, Lu G, Zhou J and Huo T: Effects of transforming growth factor $\beta-1$ infected human bone marrow mesenchymal stem cells on high- and low-metastatic potential hepatocellular carcinoma. Eur J Med Res 20: 56, 2015.

8. Zhang JW, Li Y, Zeng XC, Zhang T, Fu BS, Yi HM, Zhang Q and Jiang N: miR-630 overexpression in hepatocellular carcinoma tissues is positively correlated with alpha-fetoprotein. Med Sci Monit 21: 667-673, 2015

9. Hou YF, Wei YG, Li B, Yang JY, Wen TF, Xu MQ, Yan LN and Wang WT: Upper abdominal shape as a risk factor of extended operation time and severe postoperative complications in HCC hepatectomy through subcostal incision. World J Surg Oncol 13: 298, 2015.

10. Zhao Y, Fang Z, Luo J, Liu Q, Xu G, Pan H, Wei W and Yan Z: Evaluation of extrahepatic collateral arteries in hepatocellular carcinoma in three independent groups in a single center. Exp Ther Med 10: 2366-2374, 2015

11. Wang L, Wang J, Zhang X, Li J, Wei X, Cheng J, Ling Q, Xie H, Zhou L, Xu X and Zheng S: Diagnostic value of preoperative needle biopsy for tumor grading assessment in hepatocellular carcinoma. PLoS One 10: e0144216, 2015.

12. Selitsky SR, Baran-Gale J, Honda M, Yamane D, Masaki T, Fannin EE, Guerra B, Shirasaki T, Shimakami T, Kaneko S, et al: Small tRNA-derived RNAs are increased and more abundant than microRNAs in chronic hepatitis B and C. Sci Rep 5: 7675, 2015.

13. Zuo D, Chen L, Liu X, Wang X, Xi Q, Luo Y, Zhang N and Guo H Combination of miR-125b and miR-27a enhances sensitivity and specificity of AFP-based diagnosis of hepatocellular carcinoma. Tumour Biol 37: 6539-6549, 2016.

14. Jiang L, Cheng Q, Zhang BH and Zhang MZ: Circulating microRNAs as biomarkers in hepatocellular carcinoma screening: A validation set from China. Medicine (Baltimore) 94 e603, 2015.

15. Bartel DP: MicroRNAs: Genomics, biogenesis, mechanism, and function. Cell 116: 281-297, 2004.

16. Fornari F, Ferracin M, Trerè D, Milazzo M, Marinelli S, Galassi M, Venerandi L, Pollutri D, Patrizi C, Borghi A, et al: Circulating microRNAs, miR-939, miR-595, miR-519d and miR-494, Identify Cirrhotic Patients with HCC. PLoS One 10: e0141448, 2015

17. He R, Yang L, Lin X, Chen X, Lin X, Wei F, Liang X, Luo Y Wu Y, Gan T, et al: miR-30a-5p suppresses cell growth and enhances apoptosis of hepatocellular carcinoma cells via targeting AEG-1. Int J Clin Exp Pathol 8: 15632-15641, 2015

18. de Carvalho AC, Scapulatempo-Neto C, Maia DC, Evangelista AF, Morini MA, Carvalho AL and Vettore AL: Erratum: Accuracy of microRNAs as markers for the detection of neck lymph node metastases in patients with head and neck squamous cell carcinoma. BMC Med 13: 155, 2015.

19. de Carvalho AC, Scapulatempo-Neto C, Maia DC Evangelista AF, Morini MA, Carvalho AL and Vettore AL: Accuracy of microRNAs as markers for the detection of neck lymph node metastases in patients with head and neck squamous cell carcinoma. BMC Med 13: 108, 2015.

20. Liu H, Li W, Chen C, Pei Y and Long X: MiR-335 acts as a potential tumor suppressor miRNA via downregulating ROCK1 expression in hepatocellular carcinoma. Tumour Biol 36 6313-6319, 2015.

21. Yin J, Hou P, Wu Z, Wang T and Nie Y: Circulating miR-375 and miR-199a-3p as potential biomarkers for the diagnosis of hepatocellular carcinoma. Tumour Biol 36: 4501-4507, 2015.

22. Liu Y, Ren F, Luo Y, Rong M, Chen G and Dang Y: Down-Regulation of MiR-193a-3p Dictates Deterioration of HCC: A Clinical Real-Time qRT-PCR Study. Med Sci Monit 21: 2352-2360, 2015

23. Zhang X, Tang W, Li R, He R, Gan T, Luo Y, Chen G and Rong M: Downregulation of microRNA-132 indicates progression in hepatocellular carcinoma. Exp Ther Med 12: 2095-2101, 2016.

24. Motawi TK, Shaker OG, El-Maraghy SA and Senousy MA: Serum MicroRNAs as potential biomarkers for early diagnosis of hepatitis C Virus-related hepatocellular carcinoma in egyptian patients. PLoS One 10: e0137706, 2015.
25. Tu H, Wei G, Cai Q, Chen X, Sun Z, Cheng C, Zhang L, Feng Y, Zhou H, Zhou B and Zeng T: MicroRNA-212 inhibits hepatocellular carcinoma cell proliferation and induces apoptosis by targeting FOXA1. Onco Targets Ther 8: 2227-2235, 2015.

26. Zhang JG, Shi Y, Hong DF, Song M, Huang D, Wang CY and Zhao G: miR-148b suppresses cell proliferation and invasion in hepatocellular carcinoma by targeting WNT1/ $\beta$-catenin pathway. Sci Rep 5: 8087, 2015.

27. Guo GX,Li QY, Ma WL, Shi ZH and Ren XQ: MicroRNA-485-5p suppresses cell proliferation and invasion in hepatocellular carcinoma by targeting stanniocalcin 2. Int J Clin Exp Pathol 8: 12292-12299, 2015.

28. Liu Y, Ren F, Rong M, Luo Y, Dang Y and Chen G: Association between underexpression of microrna-203 and clinicopathological significance in hepatocellular carcinoma tissues. Cancer Cell Int 15: 62, 2015.

29. Yao H, Liu X, Chen S, Xia W and Chen X: Decreased expression of serum miR-424 correlates with poor prognosis of patients with hepatocellular carcinoma. Int J Clin Exp Pathol 8: 14830-14835, 2015.

30. Huang CS, Yu W, Cui H, Wang YJ, Zhang L, Han F and Huang T: Increased expression of miR-21 predicts poor prognosis in patients with hepatocellular carcinoma. Int J Clin Exp Pathol 8: 7234-7238, 2015

31. Chen Z, Huang Z, Ye Q, Ming Y, Zhang S, Zhao Y, Liu L, Wang Q and Cheng K: Prognostic significance and anti-proliferation effect of microRNA-365 in hepatocellular carcinoma. Int J Clin Exp Pathol 8: 1705-1711, 2015.

32. Lagos-Quintana M, Rauhut R, Yalcin A, Meyer J, Lendeckel W and Tuschl T: Identification of tissue-specific microRNAs from mouse. Curr Biol 12: 735-739, 2002.

33. Koutsoulidou A, Mastroyiannopoulos NP, Furling D, Uney JB and Phylactou LA: Expression of miR-1, miR-133a, miR-133b and miR-206 increases during development of human skeletal muscle. BMC Dev Biol 11: 34, 2011.

34. Babiarz JE, Ravon M, Sridhar S, Ravindran P, Swanson B, Bitter H, Weiser T, Chiao E, Certa U and Kolaja KL: Determination of the human cardiomyocyte mRNA and miRNA differentiation network by fine-scale profiling. Stem Cells Dev 21: 1956-1965, 2012.

35. Li Y, Cai X, Guan Y, Wang L, Wang S, Li Y, Fu Y, Gao X and Su G: Adiponectin upregulates miR-133a in cardiac hypertrophy through AMPK activation and reduced ERK1/2 phosphorylation. PLoS One 11: e0148482, 2016

36. Wang LK, Hsiao TH, Hong TM, Chen HY, Kao SH, Wang WL, Yu SL, Lin CW and Yang PC: MicroRNA-133a suppresses multiple oncogenic membrane receptors and cell invasion in non-small cell lung carcinoma. PLoS One 9: e96765, 2014.

37. Guo J, Xia B, Meng F and Lou G: miR-133a suppresses ovarian cancer cell proliferation by directly targeting insulin-like growth factor 1 receptor. Tumour Biol 35: 1557-1564, 2014.

38. Zhang W, Liu K, Liu S, Ji B, Wang Y and Liu Y: MicroRNA-133a functions as a tumor suppressor by targeting IGF-1R in hepatocellular carcinoma. Tumour Biol 36: 9779-9788, 2015.

39. Yoshino H, Chiyomaru T, Enokida H, Kawakami K, Tatarano S, Nishiyama K, Nohata N, Seki N and Nakagawa M: The tumour-suppressive function of miR-1 and miR-133a targeting TAGLN2 in bladder cancer. Br J Cancer 104: 808-818, 2011.

40. Cui W, Zhang S, Shan C, Zhou L and Zhou Z: microRNA-133a regulates the cell cycle and proliferation of breast cancer cells by targeting epidermal growth factor receptor through the EGFR/Akt signaling pathway. FEBS J 280: 3962-3974, 2013

41. Kojima S, Chiyomaru T, Kawakami K, Yoshino H, Enokida H, Nohata N, Fuse M, Ichikawa T, Naya Y, Nakagawa M and Seki N: Tumour suppressors miR-1 and miR-133a target the oncogenic function of purine nucleoside phosphorylase (PNP) in prostate cancer. Br J Cancer 106: 405-413, 2012

42. Cheng H, Fertig EJ, Ozawa H, Hatakeyama H, Howard JD, Perez J, Considine M, Thakar M, Ranaweera R, Krigsfeld G and Chung CH: Decreased SMAD4 expression is associated with induction of epithelial-to-mesenchymal transition and cetuximab resistance in head and neck squamous cell carcinoma. Cancer Biol Ther 16: 1252-1258, 2015.

43. Guo Y, Sheng Q, Li J, Ye F, Samuels DC and Shyr Y: Large scale comparison of gene expression levels by microarrays and RNAseq using TCGA data. PLoS One 8: e71462, 2013.

44. Szklarczyk D, Franceschini A, Wyder S, Forslund K, Heller D, Huerta-Cepas J, Simonovic M, Roth A, Santos A, Tsafou KP, et al: STRING v10: Protein-protein interaction networks, integrated over the tree of life. Nucleic Acids Res 43 (Database issue): D447-D452, 2015. 
45. Zhao L and Wang W: miR-125b suppresses the proliferation of hepatocellular carcinoma cells by targeting Sirtuin7. Int J Clin Exp Med 8: 18469-18475, 2015.

46. Chen Y, Dong X, Yu D and Wang X: Serum miR-96 is a promising biomarker for hepatocellular carcinoma in patients with chronic hepatitis B virus infection. Int J Clin Exp Med 8: 18462-18468, 2015.

47. Gan TQ, Tang RX, He RQ, Dang YW, Xie Y and Chen G: Upregulated MiR-1269 in hepatocellular carcinoma and its clinical significance. Int J Clin Exp Med 8: 714-721, 2015.

48. Mao B and Wang G: MicroRNAs involved with hepatocellular carcinoma (Review). Oncol Rep 34: 2811-2820, 2015.

49. Yang W, Dou C, Wang Y, Jia Y, Li C, Zheng X and Tu K: MicroRNA-92a contributes to tumor growth of human hepatocellular carcinoma by targeting FBXW7. Oncol Rep 34: 2576-2584, 2015.

50. Yang J, Liu X, Yuan X and Wang Z: miR-99b promotes metastasis of hepatocellular carcinoma through inhibition of claudin 11 expression and may serve as a prognostic marker. Oncol Rep 34: 1415-1423, 2015.

51. Jin Q, Li XJ and Cao PG: MicroRNA-26b enhances the radiosensitivity of hepatocellular carcinoma cells by targeting EphA2. Tohoku J Exp Med 238: 143-151, 2016.

52. Yang F, Li QJ, Gong ZB, Zhou L, You N, Wang S, Li XL, Li JJ, An JZ, Wang DS, et al: MicroRNA-34a targets Bcl-2 and sensitizes human hepatocellular carcinoma cells to sorafenib treatment. Technol Cancer Res Treat 13: 77-86, 2014.

53. Wu G, Wang Y, Lu X, He H, Liu H, Meng X, Xia S, Zheng K and Liu B: Low mir-372 expression correlates with poor prognosis and tumor metastasis in hepatocellular carcinoma. BMC Cancer 15: 182, 2015.

54. Drakaki A, Hatziapostolou M, Polytarchou C, Vorvis C, Poultsides GA, Souglakos J, Georgoulias V and Iliopoulos D: Functional microRNA high throughput screening reveals miR-9 as a central regulator of liver oncogenesis by affecting the PPARA-CDH1 pathway. BMC Cancer 15: 542, 2015.

55. Wang W, Wang X, Zhang Y, Wang D, Gao H, Wang L and Gao S: Prognostic role of microRNA-150 in various carcinomas: a meta-analysis. Onco Targets Ther 9: 1371-1379, 2016.

56. Mirghasemi A, Taheriazam A, Karbasy SH, Torkaman A, Shakeri M, Yahaghi E and Mokarizadeh A: Down-regulation of miR-133a and miR-539 are associated with unfavorable prognosis in patients suffering from osteosarcoma. Cancer Cell Int 15: 86, 2015.
57. Fujiwara T, Katsuda T, Hagiwara K, Kosaka N, Yoshioka Y, Takahashi RU, Takeshita F, Kubota D, Kondo T, Ichikawa $\mathrm{H}$, et al: Clinical relevance and therapeutic significance of microRNA-133a expression profiles and functions in malignant osteosarcoma-initiating cells. Stem Cells 32: 959-973, 2014.

58. Ji F, Zhang H, Wang Y, Li M, Xu W, Kang Y, Wang Z, Wang Z, Cheng P, Tong D, et al: MicroRNA-133a, downregulated in osteosarcoma, suppresses proliferation and promotes apoptosis by targeting Bcl-xL and Mcl-1. Bone 56: 220-226, 2013.

59. Lan D, Zhang X, He R, Tang R, Li P, He Q and Chen G: miR-133a is downregulated in non-small cell lung cancer: A study of clinical significance. Eur J Med Res 20: 50, 2015.

60. Akanuma N, Hoshino I, Akutsu Y, Murakami K, Isozaki Y, Maruyama T, Yusup G, Qin W, Toyozumi T, Takahashi M, et al: MicroRNA-133a regulates the mRNAs of two invadopodia-related proteins, FSCN1 and MMP14, in esophageal cancer. Br J Cancer 110: 189-198, 2014.

61. Wang G, Zhu S, Gu Y, Chen Q, Liu X and Fu H: MicroRNA-145 and microRNA-133a inhibited proliferation, migration, and invasion, while promoted apoptosis in hepatocellular carcinoma cells via targeting FSCN1. Dig Dis Sci 60: 3044-3052, 2015.

62. Lin XJ, Chong Y, Guo ZW, Xie C, Yang XJ, Zhang Q, Li SP, Xiong Y, Yuan Y, Min J, et al: A serum microRNA classifier for early detection of hepatocellular carcinoma: A multicentre, retrospective, longitudinal biomarker identification study with a nested case-control study. Lancet Oncol 16: 804-815, 2015.

63. Kaufmann R, Mussbach F, Henklein P and Settmacher U: Proteinase-activated receptor 2-mediated calcium signaling in hepatocellular carcinoma cells. J Cancer Res Clin Oncol 137: 965-973, 2011.

64. Wu R, Duan L, Cui F, Cao J, Xiang Y, Tang Y and Zhou L: S100A9 promotes human hepatocellular carcinoma cell growth and invasion through RAGE-mediated ERK1/2 and p38 MAPK pathways. Exp Cell Res 334: 228-238, 2015.

65. Huang WT, Wang HL, Yang H, Ren FH, Luo YH, Huang CQ, Liang YY, Liang HW, Chen G and Dang YW: Lower expressed miR-198 and its potential targets in hepatocellular carcinoma: A clinicopathological and in silico study. Onco Targets Ther 9: 5163-5180, 2016.

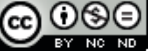

This work is licensed under a Creative Commons Attribution-NonCommercial-NoDerivatives 4.0 International (CC BY-NC-ND 4.0) License. 